T. Tsuji

Nagoya Math. J.

Vol. 55 (1974), 33-80

\title{
SIEGEL DOMAINS OVER SELF-DUAL CONES AND THEIR AUTOMORPHISMS
}

\author{
TADASHI TSUJI
}

\section{Introduction}

The Lie algebra $g_{h}$ of all infinitesimal automorphisms of a Siegel domain in terms of polynomial vector fields was investigated by Kaup, Matsushima and Ochiai [6]. It was proved in [6] that $g_{h}$ is a graded Lie algebra; $\mathfrak{g}_{h}=\mathfrak{g}_{-1}+\mathfrak{g}_{-1 / 2}+\mathfrak{g}_{0}+\mathfrak{g}_{1 / 2}+\mathfrak{g}_{1}$ and the Lie subalgebra $\mathfrak{g}_{a}$ of all infinitesimal affine automorphisms is given by the graded subalgebra; $\mathfrak{g}_{a}=\mathfrak{g}_{-1}+\mathfrak{g}_{-1 / 2}+\mathfrak{g}_{0}$. Nakajima [9] proved without the assumption of homogeneity that the non-affine parts $\mathfrak{g}_{1 / 2}$ and $\mathfrak{g}_{1}$ can be determined from the affine part $g_{a}$.

The main purpose of the present paper is to determine explicitly the Lie algebras $\mathfrak{g}_{h}$ for Siegel domains over self-dual cones. In $\S 2$ we will prove that if the adjoint representation $\rho$ of $g_{0}$ on $g_{-1}$ is irreducible, then $\mathfrak{g}_{h}$ is simple or $\mathfrak{g}_{h}=\mathfrak{g}_{a}$ (Theorem 2.1). Moreover using Nakajima's result we will give sufficient conditions of the vanishing of $g_{1 / 2}$ (Proposition 2.3 and Corollary 2.7) and a method of calculating $\mathfrak{g}_{1 / 2}$ and $\mathfrak{g}_{1}$ (Propositions 2.6 and 2.8). Using the results in $\S 2$, we determine in $\S 3$ (Theorems 3.3-3.6) infinitesimal automorphisms of most of the homogeneous Siegel domains over self-dual cones (other than circular cones) which were constructed by Pjateckii-Sapiro [10].

The circular cone $C(n)$ of dimension $n(n \geq 3)$ is defined to be the set $\left\{t\left(x_{1}, x_{2}, \cdots, x_{n}\right) \in \boldsymbol{R}^{n} ; x_{1}>0, x_{1} x_{2}-x_{3}^{2}-\cdots-x_{n}^{2}>0\right\}$. Pjateckii-Sapiro [10] found all the homogeneous Siegel domains over circular cones which are constructed by using the representation theory of Clifford algebras. But it was shown by Kaneyuki and Tsuji [5] that there exists a homogeneous Siegel domain over a circular cone which does not appear in Pjateckii-Sapiro's construction. In view of this fact the purpose in $\S 4$ is to give a method of constructing all homogeneous Siegel domains over

Received January 21, 1974. 
circular cones (Theorem 4.4) by making use of the considerations analogous to [5].

Pjateckii-Sapiro [10] pointed out without proof that the exceptional bounded symmetric domain in $C^{16}$ is realized as a Siegel domain over the cone $C$ (8). In $\S 5$ we consider a certain homogeneous Siegel domain $D$ over $C$ (8), which is implicitly given in [10], and by means of results in $\S 2$ and $\S 4$ we prove that $D$ is isomorphic to the above exceptional symmetric domain (Theorem 5.4).

Finally, in $\S 6$ we determine infinitesimal automorphisms of homogeneous Siegel domains over circular cones (Theorem 6.1, Propositions 6.2 and 6.3).

Some of results of the present paper were announced in the note [15].

The author wishes to express his hearty thanks to Prof. S. Kaneyuki for his helpful suggestions and encouragement during the preparation of this paper.

\section{§ 1. Preliminaries}

In this section, after introducing notations which are used throughout this paper, we recall some of results of [6] and [9].

1.1. Let $R$ be a real vector space of dimension $n$ and $W$ be a complex vector space of dimension $m$. Let $D(V, F)$ denote a Siegel domain of type I or type II in $R^{c} \times W$ associated with a convex cone $V$ in $R$ and a $V$-hermitian form $F$ on $W$, which is defined by PjateckiiSapiro [10], where $R^{c}$ is the complexification of $R$. Throughout this paper we will employ the following notations;

$\mathfrak{g}_{h}$ (resp. $g_{a}$ ); the Lie algebra of all infinitesimal holomorphic (resp. affine) automorphisms of $D(V, F)$.

$g(V)$; the Lie algebra of the automorphism group $G(V)=\{g \in G L(R)$; $g V=V\}$ of the cone $V$.

$\left\{e_{1}, \cdots, e_{n}\right\}$ (resp. $\left\{f_{1}, \cdots, f_{m}\right\}$ ); a base of $R$ (resp. $W$ ).

$\left(z_{1}, \cdots, z_{n}, w_{1}, \cdots, w_{m}\right)$; the complex coordinate system of $R^{c} \times W$ associated with the base $\left\{e_{1}, \cdots, e_{n}, f_{1}, \cdots, f_{m}\right\}$.

The following ranges of indices will be taken in each summation: $1 \leq j, k$, $l, \cdots \leq n, 1 \leq \alpha, \beta, \gamma, \cdots \leq m$.

For a positive integer $p, U(p)$ (resp. $O(p)$ ) denotes the unitary (resp.-real orthogonal) group of degree $p$ and $E_{p}$ denotes the unit matrix of degree $p$. And for two positive integers $p$ and $q$, we denote by $M(p, q ; F)$ the 
real (resp. complex) vector space of all real (resp. complex) $p \times q$ matrices and by $\mathfrak{g l}(p, \boldsymbol{F})$ the real (resp. complex) general linear Lie algebra of degree $p$, where $\boldsymbol{F}=\boldsymbol{R}$ (resp. $\boldsymbol{C}$ ).

1.2. Put $\partial=\sum z_{k} \partial / \partial z_{k}+\frac{1}{2} \sum w_{\alpha} \partial / \partial w_{\alpha}$ and $\partial^{\prime}=i \sum w_{\alpha} \partial / \partial w_{\alpha}$. Then the following results (1.4)-(1.6) are known in [6].

(1.1) The vector field $\partial$ belongs to $\mathfrak{g}_{h}$ and $\mathfrak{g}_{h}$ is a graded Lie algebra; $\mathfrak{g}_{h}=\mathfrak{g}_{-1}+\mathfrak{g}_{-1 / 2}+\mathfrak{g}_{0}+\mathfrak{g}_{1 / 2}+\mathfrak{g}_{1}$, where $\mathfrak{g}_{\lambda}$ is the $\lambda$-eigenspace of $\operatorname{ad}(\partial)$ $\left(\lambda= \pm 1, \pm \frac{1}{2}, 0\right)$. Furthermore $g_{a}$ is the graded subalgebra; $g_{a}=$ $\mathfrak{g}_{-1}+\mathfrak{g}_{-1 / 2}+\mathfrak{g}_{0}$.

$$
\mathfrak{g}_{-1}=\left\{\sum a^{k} \partial / \partial z_{k} ; a^{k} \in \boldsymbol{R}\right\} \text {. }
$$

(1.3) $\mathfrak{g}_{-1 / 2}=\left\{2 i \sum F^{k}(w, c) \partial / \partial z_{k}+\sum c^{\alpha} \partial / \partial w_{\alpha} ; c=\sum c^{\alpha} f_{\alpha} \in W\right\}$, where $F(w, c)=\sum F^{k}(w, c) e_{k}$.

$\mathfrak{g}_{0}=\left\{\sum a_{k l} z_{l} \partial / \partial z_{k}+\sum b_{\alpha \beta} w_{\beta} \partial / \partial w_{\alpha} ; A=\left(a_{k l}\right) \in \mathfrak{g}(V), B=\left(b_{\alpha \beta}\right) \in \mathfrak{g l}(W)\right.$, $A F(u, u)=F(B u, u)+F(u, B u)$ for each $u \in W\}$.

Let $\mathfrak{x}$ be the radical of $g_{h}$. Then

(1.5) $\mathfrak{x}$ is a graded ideal of $\mathfrak{g}_{h}$ such that $\mathfrak{x}=\mathfrak{x}_{-1}+\mathfrak{x}_{-1 / 2}+\mathfrak{r}_{0}$, where $\mathfrak{r}_{-2}=\mathfrak{x} \cap \mathfrak{g}_{-2}\left(\lambda=1, \frac{1}{2}, 0\right)$.

$$
\operatorname{dim} \mathfrak{g}_{\lambda}=\operatorname{dim} \mathfrak{g}_{-\lambda}-\operatorname{dim} \mathfrak{r}_{-\lambda}\left(\lambda=1, \frac{1}{2}\right) .
$$

Considering (1.1) we denote by $\rho$ (resp. $\sigma$ ) the adjoint representation of the subalgebra $\mathfrak{g}_{0}$ on $\mathfrak{g}_{-1}$ (resp. $\mathfrak{g}_{-1 / 2}$ ). Let us define real linear isomorphisms $\varphi_{-1}$ and $\varphi_{-1 / 2}$ as follows;

$$
\begin{aligned}
& \varphi_{-1}: a=\sum a^{k} e_{k} \in R \mapsto \varphi_{-1}(a)=\sum a^{k} \partial / \partial z_{k} \in \mathfrak{g}_{-1}, \\
& \varphi_{-1 / 2}: c=\sum c^{\alpha} f_{\alpha} \in W \mapsto \varphi_{-1 / 2}(c)=2 i \sum F^{k}(w, c) \partial / \partial z_{k}+\sum c^{\alpha} \partial / \partial w_{\alpha} \in \mathfrak{g}_{-1 / 2} .
\end{aligned}
$$

Then by easy computations we can see that the following (1.7) and (1.8) are valid; for $a \in R, c, c^{\prime} \in W$ and $X=\sum a_{k l} z_{l} \partial / \partial z_{k}+\sum b_{\alpha \beta} w_{\beta} \partial / \partial w_{\alpha} \in \mathfrak{g}_{0}$,

$$
\rho(X)\left(\varphi_{-1}(a)\right)=-\varphi_{-1}(A a) \text { and } \sigma(X)\left(\varphi_{-1 / 2}(c)\right)=-\varphi_{-1 / 2}(B c) \text {, }
$$
where $A=\left(a_{k l}\right)$ and $B=\left(b_{\alpha \beta}\right)$. In particular $\sigma\left(\partial^{\prime}\right)\left(\varphi_{-1 / 2}(c)\right)=-\varphi_{-1 / 2}(i c)$.

$$
\left[\varphi_{-1 / 2}(c), \varphi_{-1 / 2}\left(c^{\prime}\right)\right]=4 \varphi_{-1}\left(\operatorname{Im} F\left(c^{\prime}, c\right)\right) .
$$

By the facts stated above we can identify $\rho\left(g_{0}\right)$ with a subalgebra of $g(V)$.

The following results (1.9) and (1.10) are due to Nakajima (Proposition 2.6 in [9]). 
(1.9) The subspace $g_{1 / 2}$ of $g_{h}$ consists of all polynomial vector fields $X=\sum p_{1,1}^{k} \partial / \partial z_{k}+\sum\left(p_{1,0}^{\alpha}+p_{0,2}^{\alpha}\right) \partial / \partial w_{\alpha}$ satisfying the condition $\left[\mathfrak{g}_{-1 / 2}, X\right] \subset \mathfrak{g}_{0}$, where $p_{\lambda, \mu}^{k}$ and $p_{\lambda, \mu}^{\alpha}$ are polynomials of homogeneous degree $\lambda$ in $z_{1}, \cdots, z_{n}$ and homogeneous degree $\mu$ in $w_{1}, \cdots, w_{m}$.

(1.10) The subspace $g_{1}$ of $g_{h}$ consists of all polynomial vector fields $X=\sum p_{2,0}^{k} \partial / \partial z_{k}+\sum p_{1,1}^{\alpha} \partial / \partial w_{\alpha}$ satisfying the following conditions; $\left[\mathfrak{g}_{-1 / 2}, X\right] \subset \mathfrak{g}_{1 / 2},\left[\mathfrak{g}_{-1}, X\right] \subset \mathfrak{g}_{0}$ and $\operatorname{Im} \operatorname{Tr} \sigma([Y, X])=0$ for each $Y \in \mathfrak{g}_{-1}$.

\section{§ 2. Lie algebras of infinitesimal automorphisms}

2.1. Kaneyuki and Sudo [4] proved that if $D(V, F)$ is an irreducible symmetric domain (or equivalently $\mathfrak{g}_{h}$ is simple), then the representation $\rho$ is irreducible. Conversely without the assumption of homogeneity of $D(V, F)$ we have

THEOREM 2.1. If the representation $\rho$ is irreducible, then $g_{h}$ is simple or $\mathrm{g}_{h}=\mathfrak{g}_{a}$.

Proof. By our assumption we have $\mathfrak{x}_{-1}=(0)$ or $\mathfrak{x}_{-1}=\mathfrak{g}_{-1}$, since $\mathfrak{x}_{-1}$ is a subspace of $\mathfrak{g}_{-1}$ invariant under $\rho\left(\mathfrak{g}_{0}\right)$. First we suppose $\mathfrak{x}_{-1}=(0)$. Then it follows from (1.5), (1.7) and (1.8) that $\mathfrak{x}_{-1 / 2}=\mathfrak{x}_{0}=(0)$ and $\mathfrak{x}=(0)$ (this fact was proved more generally in [9]). So $\mathfrak{g}_{h}$ is semi-simple. Suppose that $g_{h}$ is not simple. Then the Siegel domain $D(\dot{V}, F)$ is reducible and the cone $V$ is decomposed into irreducible factors (cf. [9], Corollaries 4.8 and 4.9), which means that $\rho$ is not irreducible. This contradicts to our assumption. Thus $g_{h}$ is simple.

Now we consider the case $\mathfrak{r}_{-1}=g_{-1}$. It follows from (1.6) that $g_{1}$ $=(0)$. We will show that $g_{1 / 2}=(0)$. By (1.9) every $X \in g_{1 / 2}$ is represented as $X=\sum p_{1,1}^{k} \partial / \partial z_{k}+\sum\left(p_{1,0}^{\alpha}+p_{0,2}^{\alpha}\right) \partial / \partial w_{\alpha}$. Put $Z=\left[X,\left[\partial^{\prime}, X\right]\right]$. Then from the direct verification it follows that $Z$ is represented as

$$
Z=2 i \sum p_{1,0}^{\alpha} \frac{\partial p_{1,1}^{k}}{\partial w_{\alpha}} \partial / \partial z_{k}+2 i \sum\left(p_{1,0}^{\beta} \frac{\partial p_{0,2}^{\alpha}}{\partial w_{\beta}}-p_{1,1}^{k} \frac{\partial p_{1,0}^{\alpha}}{\partial z_{k}}\right) \partial / \partial w_{\alpha}
$$

By (1.1) and the fact $\partial^{\prime} \in g_{0}$, the vector field $Z$ belongs to $g_{1}=(0)$. Hence we have

$$
\sum p_{1,0}^{\alpha} \frac{\partial p_{1,1}^{k}}{\partial w_{\alpha}}=0 \quad(1 \leq k \leq n)
$$


Since $\left[\mathfrak{g}_{-1}, X\right] \subset g_{-1 / 2}$, there exist $c_{l}=\sum c_{l}^{\alpha} f_{\alpha} \in W(1 \leq l \leq n)$ such that $\left[\partial / \partial z_{l}, X\right]=2 i \sum F^{k}\left(w, c_{l}\right) \partial / \partial z_{k}+\sum c_{l}^{\alpha} \partial / \partial w_{\alpha}(1 \leq l \leq n)$. On the other hand, $\left[\partial / \partial z_{l}, X\right]=\sum \frac{\partial p_{1,1}^{k}}{\partial z_{l}} \partial / \partial z_{k}+\sum \frac{\partial p_{1,0}^{\alpha}}{\partial z_{l}} \partial / \partial w_{\alpha}(1 \leq l \leq n)$, which implies $\frac{\partial p_{1,1}^{k}}{\partial z_{l}}=2 i F^{k}\left(w, c_{l}\right)$ and $\frac{\partial p_{1,0}^{\alpha}}{\partial z_{l}}=c_{l}^{\alpha}$. Hence we have

$$
p_{1,1}^{k}=2 i \sum F^{k}\left(w, c_{l}\right) z_{l} \quad \text { and } \quad p_{1,0}^{\alpha}=\sum c_{l}^{\alpha} z_{l} \quad(1 \leq k \leq n, 1 \leq \alpha \leq \mathrm{m}) .
$$

In view of (2.1) we obtain $\sum F^{k}\left(c_{j}, c_{l}\right) z_{j} z_{l}=0(1 \leq k \leq n)$. So we get $F^{k}\left(c_{l}, c_{l}\right)=0 \quad(1 \leq k, l \leq n)$. Therefore $c_{l}=0$ and $p_{1,1}^{k}=p_{1,0}^{\alpha}=0 \quad(1 \leq k$ $\leq n, 1 \leq \alpha \leq m)$. Thus $X$ is written as $X=\sum p_{0,2}^{\alpha} \partial / \partial w_{\alpha}$. It is easily seen that $\left[\partial^{\prime}, X\right]=i X$. So both $X$ and $i X$ are contained in $g_{h}$. This means $X=0$ by the well-known theorem of $\mathrm{H}$. Cartan. Consequently we have $g_{1 / 2}=(0)$ and by (1.1) we conclude that $g_{h}=g_{a}$. q.e.d.

The above theorem will be used to determine the Lie algebras $g_{h}$ of certain Siegel domains in the following sections.

A Siegel domain $D(V, F)$ in $R^{c} \times W$ is said to be non-degenerate if the linear closure of the set $\{F(u, u) ; u \in W\}$ in $R$ coincides with $R$ (cf. [4]). Otherwise $D(V, F)$ is called degenerate.

Without the assumptions of irreducibility of $\rho$ and homogeneity of $D(V, F)$, we have

Proposition 2.2. If $D(V, F)$ is non-degenerate and $\mathrm{g}_{1 / 2}=(0)$, then $\mathfrak{g}_{h}=\mathfrak{g}_{a}$.

Proof. From (1.7) and (1.8) it follows that $D(V, F)$ is non-degenerate if and only if $\left[\mathfrak{g}_{-1 / 2}, \mathfrak{g}_{-1 / 2}\right]=\mathfrak{g}_{-1}$. For $X \in \mathfrak{g}_{1}$, we have $\left[X, \mathfrak{g}_{-1 / 2}\right] \subset \mathfrak{g}_{1 / 2}=(0)$ and so $\left[X, \mathfrak{g}_{-1}\right]=\left[X,\left[\mathfrak{g}_{-1 / 2}, \mathfrak{g}_{-1 / 2}\right]\right]=(0)$. On the other hand, the condition $\left[X, \mathfrak{g}_{-1}\right]=(0)$ implies $X=0$ (see [9], Lemma 3.1). By (1.1) we have $\mathfrak{g}_{h}$ $=\mathfrak{g}_{a}$.

q.e.d.

2.2. We now discuss sufficient conditions of the vanishing of $g_{1 / 2}$ of a Siegel domain $D(V, F)$ of type II in $R^{c} \times W$. Let $X=\sum p_{1,1}^{k} \partial / \partial z_{k}+$ $\sum\left(p_{1,0}^{\alpha}+p_{0,2}^{\alpha}\right) \partial / \partial w_{\alpha}$ be a polynomial vector field on $R^{c} \times W$. Then it is known in [9] that $X$ is contained in $g_{1 / 2}$ if and only if there exist $c_{l}=$ $\sum c_{l}^{\alpha} f_{\alpha} \in W(1 \leq l \leq n)$ and $b_{\beta \gamma}^{\alpha} \in C\left(b_{\beta \gamma}^{\alpha}=b_{\gamma \beta}^{\alpha}, 1 \leq \alpha, \beta, \gamma \leq m\right)$ satisfying the following (2.2), (2.3) and (2.4) (see (3.2) and (3.5) in [9]);

(2.2) $X$ is represented as

$$
X=2 i \sum F^{k}\left(w, c_{l}\right) z_{l} \partial / \partial z_{k}+\sum c_{l}^{\alpha} z_{l} \partial / \partial w_{\alpha}+\sum b_{\beta \gamma}^{\alpha} w_{\beta} w_{\gamma} \partial / \partial w_{\alpha} .
$$




$$
\sum_{\alpha} b_{\beta r}^{\alpha} F_{\alpha \delta}^{k}=i \sum_{\alpha, l}\left(F_{\beta \delta}^{l} \bar{c}_{l}^{\alpha} F_{\gamma \alpha}^{k}+F_{\gamma \delta}^{l} \bar{c}_{l}^{\alpha} F_{\beta \alpha}^{k}\right)
$$

for $1 \leq k \leq n, 1 \leq \beta, \gamma, \delta \leq m$, where $F_{\alpha \beta}^{k}=F^{k}\left(f_{\alpha}, f_{\beta}\right)$.

(2.4) For each $d \in W$, the matrix $A(d)=\left(A(d)_{k l}\right)$ belongs to $\mathfrak{g}(V)$, where $A(d)_{k l}=\operatorname{Im} F^{k}\left(c_{l}, d\right)$.

Proposition 2.3. If a vector field $X \in \mathfrak{g}_{1 / 2}$ satisfies the condition $\rho\left(\left[g_{-1 / 2}, X\right]\right)=(0)$, then $X=0$.

Proof. By (2.2) there exist $c_{l} \in W(1 \leq l \leq n)$ and $b_{\beta \gamma}^{\alpha} \in C(1 \leq \alpha, \beta, \gamma \leq m)$ such that $X$ is represented as $X=2 i \sum F^{k}\left(w, c_{l}\right) z_{l} \partial / \partial z_{k}+\sum c_{l}^{\alpha} z_{l} \partial / \partial w_{\alpha}+$ $\sum b_{\beta \gamma}^{\alpha} w_{\beta} w_{\gamma} \partial / \partial w_{\alpha}$. For each $d \in W$, we can verify that the matrix $\rho\left(\left[\varphi_{-1 / 2}(d), X\right]\right)$ coincides with $\left(4 \operatorname{Im} F^{k}\left(c_{l}, d\right)\right)$. From our assumption it follows that $F^{k}\left(c_{l}, d\right)=0$ for every $d \in W(1 \leq k, l \leq n)$. Therefore $c_{l}=$ $0(1 \leq l \leq n)$ and $X$ is written as $X=\sum p_{0,2}^{\alpha} \partial / \partial w_{\alpha}$. By the same consideration as in the proof of Theorem 2.1 we have $X=0$. q.e.d.

Now we suppose that $W$ is the direct sum of subspaces $W_{i}(i=1,2)$ satisfying the condition $\boldsymbol{F}\left(W_{1}, W_{2}\right)=(0)$. Let $\boldsymbol{F}_{i}$ denote the restriction of the $V$-hermitian form $F$ to $W_{i} \times W_{i}$. Then $F_{i}$ is a $V$-hermitian form on $W_{i}$. We denote by $\mathfrak{g}_{h}^{(i)}=\mathfrak{g}_{-1}^{(i)}+\mathfrak{g}_{-1 / 2}^{(i)}+\mathfrak{g}_{0}^{(i)}+\mathfrak{g}_{1 / 2}^{(i)}+\mathfrak{g}_{1}^{(i)}$ the Lie algebra of all infinitesimal automorphisms of the Siegel domain $D\left(V, F_{i}\right)$ in $R^{c} \times W_{i}$. We can assume that $\left\{f_{1}, \cdots, f_{m_{1}}\right\}$ (resp. $\left\{f_{m_{1}+1}, \cdots, f_{m}\right\}$ ) is a base of $W_{1}$ (resp. $\left.W_{2}\right)$, where $m_{1}=\operatorname{dim} W_{1}$.

We define a linear map $\Phi$ of the Lie algebra of all polynomial vector fields on $R^{c} \times W$ into that of all polynomial vector fields on $R^{c} \times W_{1}$ by

$$
\begin{aligned}
& \Phi\left(\sum_{1 \leq k \leq n} p_{\lambda, \mu}^{k} \partial / \partial z_{k}\right)=\sum_{1 \leq k \leq n}\left(p_{\lambda, \mu}^{k} \circ \iota\right) \partial / \partial z_{k}, \\
& \Phi\left(\sum_{1 \leq \alpha \leq m} p_{\lambda, \mu}^{\alpha} \partial / \partial w_{\alpha}\right)=\sum_{1 \leq \alpha \leq m_{1}}\left(p_{\lambda, \mu}^{\alpha} \circ \iota\right) \partial / \partial w_{\alpha},
\end{aligned}
$$

where $\iota$ is the injection $\left(z, w_{1}\right) \in R^{c} \times W_{1} \mapsto\left(z, w_{1}+0\right) \in R^{c} \times W$.

For

$$
X=2 i \sum F^{k}\left(w, c_{l}\right) z_{l} \partial / \partial z_{k}+\sum c_{l}^{\alpha} z_{l} \partial / \partial w_{\alpha}+\sum b_{\beta \gamma}^{\alpha} w_{\beta} w_{r} \partial / \partial w_{\alpha} \in \mathfrak{g}_{1 / 2}
$$

(cf. (2.2)), we define two vector fields $X^{(1)}$ and $X^{(2)}$ by

$$
X^{(1)}=2 i \sum F_{1}^{k}\left(w_{1}, c_{l, 1}\right) z_{l} \partial / \partial z_{k}+\sum_{1 \leq \alpha \leq m_{1}} c_{l}^{\alpha} z_{l} \partial / \partial w_{\alpha}
$$




$$
\begin{aligned}
& +\sum_{1 \leq \alpha, \beta, \gamma \leq m_{1}} b_{\beta \gamma}^{\alpha} w_{\beta} w_{r} \partial / \partial w_{\alpha}, \\
X^{(2)}= & 2 i \sum F_{2}^{k}\left(w_{2}, c_{l, 2}\right) z_{l} \partial / \partial z_{k}+\sum_{m_{1}<\alpha \leq m} c_{l}^{\alpha} z_{l} \partial / \partial w_{\alpha} \\
& +\sum_{m_{1}<\alpha, \beta, \gamma \leq m} b_{\beta \gamma}^{\alpha} w_{\beta} w_{\gamma} \partial / \partial w_{\alpha},
\end{aligned}
$$

where $w=w_{1}+w_{2}, c_{l}=c_{l, 1}+c_{l, 2} \in W=W_{1}+W_{2}$. Then we get

LEMMA 2.4. For each $X \in \mathfrak{g}_{1 / 2}, X^{(i)}$ belongs to $\mathfrak{g}_{1 / 2}^{(i)}(i=1,2)$ and $\Phi(X)$ $=X^{(1)}$.

Proof. We will show that the polynomial vector field $X^{(1)}$ (resp. $X^{(2)}$ ) on $R^{c} \times W_{1}$ (resp. $R^{c} \times W_{2}$ ) satisfies the conditions (2.2), (2.3) and (2.4). In fact, by (2.6) $X^{(1)}$ (resp. $X^{(2)}$ ) satisfies the condition (2.2). By using the equalities $F\left(W_{1}, W_{2}\right)=(0), F_{1}^{k}\left(f_{\alpha}, f_{\beta}\right)=F_{\alpha \beta}^{k}\left(1 \leq \alpha, \beta \leq m_{1}\right), \quad F_{2}^{k}\left(f_{\alpha}, f_{\beta}\right)$ $=F_{\alpha \beta}^{k}\left(m_{1}<\alpha, \beta \leq m\right)$ and the fact $X \in \mathfrak{g}_{1 / 2}$, we have

$$
\begin{aligned}
\sum_{1 \leq \alpha \leq m_{1}} b_{\beta \gamma}^{\alpha} F_{\alpha \delta}^{k}= & \sum_{1 \leq \alpha \leq m} b_{\beta \gamma}^{\alpha} F_{\alpha \delta}^{k}=i \sum_{\substack{1 \leq l \leq n \\
1 \leq \alpha \leq m}}\left(F_{\beta \delta}^{l} \bar{c}_{l}^{\alpha} F_{\gamma \alpha}^{k}+F_{\gamma \delta}^{l} \bar{c}_{l}^{\alpha} F_{\beta \alpha}^{k}\right) \\
= & i \sum_{\substack{1 \leq l \leq n \\
1 \leq \alpha \leq m_{1}}}\left(F_{\beta \delta}^{l} \bar{c}_{l}^{\alpha} F_{\gamma \alpha}^{k}+F_{\gamma \delta}^{l} \bar{c}_{l}^{\alpha} F_{\beta \alpha}^{k}\right) \\
& \left(1 \leq k \leq n, 1 \leq \beta, \gamma, \delta \leq m_{1}\right),
\end{aligned}
$$

which implies that $X^{(1)}$ satisfies the condition (2.3). For each $d_{1} \in W_{1}$ the matrix (Im $F_{1}^{k}\left(c_{l, 1}, d_{1}\right)$ ) belongs to $g(V)$, since the matrix $\left(\operatorname{Im} F^{k}\left(c_{l}, d_{1}\right)\right)$ belongs to $\mathrm{g}(V)$ and $F^{k}\left(c_{l}, d_{1}\right)=F_{1}^{k}\left(c_{l, 1}, d_{1}\right)$. Thus we showed that $X^{(1)}$ satisfies the condition (2.4). Therefore $X^{(1)}$ is contained in $\mathfrak{g}_{1 / 2}^{(1)}$. Analogously we can see that $X^{(2)}$ belongs to $g_{1 / 2}^{(2)}$. From (2.5), (2.6) and the condition $F\left(W_{1}, W_{2}\right)=(0)$ it follows immediately that $\Phi(X)=X^{(1)}$.

q.e.d.

LEMMA 2.5. For each $X \in \mathfrak{g}_{0}, \Phi(X)$ belongs to $\mathrm{g}_{0}^{(1)}$.

Proof. We put $\sigma(X)=\left(\begin{array}{ll}\sigma_{1}(X) & \sigma_{3}(X) \\ \sigma_{2}(X) & \sigma_{4}(X)\end{array}\right)$, where $\sigma_{1}(X)$ is the submatrix of degree $m_{1}$. Then it can be easily seen that $\Phi(X)$ is represented by

$$
\Phi(X)=\sum_{1 \leq k, l \leq n} a_{k l} z_{l} \partial / \partial z_{k}+\sum_{1 \leq \alpha, \beta \leq m_{1}} b_{\alpha \beta} w_{\beta} \partial / \partial w_{\alpha},
$$

where the matrices $\left(a_{k l}\right)$ and $\left(b_{\alpha \beta}\right)$ coincide with $\rho(X)$ and $\sigma_{1}(X)$, respectively. From the condition $F\left(W_{1}, W_{2}\right)=(0)$ and (1.4) it follows that for each $u_{1} \in W_{1}$,

$$
\rho(X) F_{1}\left(u_{1}, u_{1}\right)=\rho(X) F\left(u_{1}, u_{1}\right)
$$




$$
\begin{aligned}
& =F\left(\sigma(X) u_{1}, u_{1}\right)+F\left(u_{1}, \sigma(X) u_{1}\right) \\
& =F\left(\sigma_{1}(X) u_{1}+\sigma_{2}(X) u_{1}, u_{1}\right)+F\left(u_{1}, \sigma_{1}(X) u_{1}+\sigma_{2}(X) u_{1}\right) \\
& =F_{1}\left(\sigma_{1}(X) u_{1}, u_{1}\right)+F_{1}\left(u_{1}, \sigma_{1}(X) u_{1}\right) .
\end{aligned}
$$

So, by (1.4) $\Phi(X)$ belongs to $g_{0}^{(1)}$.

q.e.d.

We now denote by $\Phi_{2}$ the map $\Phi$ restricted to the subspace $g_{2}$ of $g_{h}$ $\left(\lambda= \pm 1, \pm \frac{1}{2}, 0\right)$. Then we have

Proposition 2.6. If $\mathfrak{g}_{1 / 2}^{(2)}=(0)$, then the map $\Phi$ induces a gradepreserving linear map of $\mathfrak{g}_{h}$ into $\mathfrak{g}_{h}^{(1)}$ satisfying the following conditions:

(1) The subspace $\mathrm{g}_{-1}$ of $\mathrm{g}_{h}$ coincides with $\mathrm{g}_{-1}^{(1)}$ and $\Phi_{-1}$ is an identity. Furthermore $\Phi_{-1 / 2}$ is a surjection of $\mathfrak{g}_{-1 / 2}$ onto $\mathfrak{g}_{-1 / 2}^{(1)}$.

(2) The map $\Phi_{1 / 2}$ is an injection of $\mathrm{g}_{1 / 2}$ into $\mathrm{g}_{1 / 2}^{(1)}$.

(3) The subspace $g_{1}$ of $g_{h}$ is contained in $g_{1}^{(1)}$ and $\Phi_{1}$ is an identity.

(4) The maps $\Phi_{\lambda}$ satisfy the condition; $\Phi_{0}([X, Y])=\left[\Phi_{-\lambda}(X), \Phi_{\lambda}(Y)\right]$ for $X \in \mathfrak{g}_{-\lambda}, Y \in \mathfrak{g}_{\lambda}\left(\lambda=1, \frac{1}{2}\right)$.

Proof. By (1.2) it is obvious that $g_{-1}=g_{-1}^{(1)}$ and $\Phi_{-1}\left(\partial / \partial z_{k}\right)=\partial / \partial z_{k}$. Now we show $\Phi\left(g_{-1 / 2}\right)=g_{-1 / 2}^{(1)}$. In fact, from (1.3) and the condition $F\left(W_{1}, W_{2}\right)=(0)$ it follows that $\Phi\left(\varphi_{-1 / 2}(c)\right)=\varphi_{-1 / 2}\left(c_{1}\right)$ for $c=c_{1}+c_{2} \in W=$ $W_{1}+W_{2}$. Thus we have $\Phi\left(\mathfrak{g}_{-1 / 2}\right)=\mathfrak{g}_{-1 / 2}^{(1)}$ and the assertion (1) was proved.

By Lemma 2.4 we have $\Phi\left(g_{1 / 2}\right) \subset g_{1 / 2}^{(1)}$. For $X \in g_{1 / 2}$ we suppose that $\Phi_{1 / 2}(X)=0$. Then from the assumption $g_{1 / 2}^{(2)}=(0)$ and Lemma 2.4 it follows that $X^{(1)}=X^{(2)}=0$ and $X$ is represented as $X=\sum p_{0,2}^{\alpha} \partial / \partial w_{\alpha}$. Therefore, (as we stated before,) $X=0$. Thus the assertion (2) was proved.

Now we show that $\Phi_{1}(X)=X$ for each $X \in g_{1}$. In fact, let $X=$ $\sum A_{j l}^{k} z_{j} z_{l} \partial / \partial z_{k}+\sum B_{l \beta}^{\alpha} z_{l} w_{\beta} \partial / \partial w_{\alpha} \in g_{1}\left(A_{j l}^{k}=A_{l j}^{k}, B_{l \beta}^{\alpha} \in C\right.$, cf. (1.10)). Then from the condition $\left[\mathrm{g}_{-1 / 2}, X\right] \subset g_{1 / 2}$ it follows that for each $c \in W$,

$$
\begin{aligned}
{\left[\varphi_{-1 / 2}(c), X\right]=} & 2 i \sum\left(2 F^{j}(w, c) A_{j l}^{k}-B_{l \beta}^{\alpha} F^{k}\left(f_{\alpha}, c\right) w_{\beta}\right) z_{l} \partial / \partial z_{k} \\
& +\sum c^{\beta} B_{l \beta}^{\alpha} z_{l} \partial / \partial w_{\alpha}+2 i \sum B_{k \beta}^{\alpha} F^{k}(w, c) w_{\beta} \partial / \partial w_{\alpha}
\end{aligned}
$$

belongs to $g_{1 / 2}$. On the other hand, by (2.2) there exist $c_{l} \in W(1 \leq l \leq n)$ and $b_{\beta r}^{\alpha} \in C(1 \leq \alpha, \beta, \gamma \leq m)$ such that

$$
\left[\varphi_{-1 / 2}(c), X\right]=2 i \sum F^{k}\left(w, c_{l}\right) z_{l} \partial / \partial z_{k}+\sum c_{l}^{\alpha} z_{l} \partial / \partial w_{\alpha}+\sum b_{\beta \gamma}^{\alpha} w_{\beta} w_{\gamma} \partial / \partial w_{\alpha} .
$$

By the assumption $\mathrm{g}_{1 / 2}^{(2)}=(0)$ and Lemma 2.4 we have $\left[\varphi_{-1 / 2}(c), X\right]^{(2)}=0$. Therefore by (2.6) $c_{l}$ is contained in $W_{1}$ (i.e., $c_{l}^{\alpha}=0$ if $m_{1}<\alpha \leq m$ ). 
By (2.7) we have

$$
B_{l \beta}^{\alpha}=0\left(1 \leq l \leq n, m_{1}<\alpha \leq m, 1 \leq \beta \leq m\right)
$$

and

$$
F^{k}\left(w_{1}, c_{l}\right)=2 \sum_{1 \leq j \leq n} F^{j}(w, c) A_{j l}^{k}-\sum_{\substack{1 \leq \alpha \leq m_{1} \\ 1 \leq \beta \leq m}} B_{l \beta}^{\alpha} F^{k}\left(f_{\alpha}, c\right) w_{\beta} .
$$

By the condition $F\left(W_{1}, W_{2}\right)=(0)$ we get

$$
2 \sum_{1 \leq j \leq n} F^{j}\left(w_{2}, c_{2}\right) A_{j l}^{k}-\sum_{\substack{1 \leq \alpha \leq m_{1} \\ m_{1}<\beta \leq m}} B_{l \beta}^{\alpha} F^{k}\left(f_{\alpha}, c_{1}\right) w_{\beta}=0 .
$$

As $c=c_{1}+c_{2}$ is an arbitrary element in $W=W_{1}+W_{2}$, so

$$
\sum_{\substack{1 \leq \alpha \leq m_{1} \\ m_{1}<\beta \leq m}} B_{l \beta}^{\alpha} F^{k}\left(f_{\alpha}, c_{1}\right) w_{\beta}=0
$$

By putting $c_{1}=\sum_{1 \leq \alpha \leq m_{1}} B_{l \beta}^{\alpha} f_{\alpha}$ we have $F^{k}\left(\sum_{1 \leq \alpha \leq m_{1}} B_{l \beta}^{\alpha} f_{\alpha}, \sum_{1 \leq \alpha \leq m_{1}} B_{l \beta}^{\alpha} f_{\alpha}\right)=0$.

Therefore

$$
B_{l \beta}^{\alpha}=0 \quad\left(1 \leq l \leq n, 1 \leq \alpha \leq m_{1}<\beta \leq m\right),
$$

and $X$ is written as

$$
X=\sum_{1 \leq j, k, l \leq n} A_{j l}^{k} z_{j} z_{l} \partial / \partial z_{k}+\sum_{\substack{1 \leq l \leq n \\ 1 \leq \alpha, \beta \leq m_{1}}} B_{l \beta}^{\alpha} z_{l} w_{\beta} \partial / \partial w_{\alpha} .
$$

By (2.5) we conclude that $\Phi_{1}(X)=X$.

We want to show $g_{1} \subset g_{1}^{(1)}$. It is enough to show that each element $X \in \mathfrak{g}_{1}$ considered as a polynomial vector field on $R^{c} \times W_{1}$ satisfies the conditions in (1.10).

For each $c_{1} \in W_{1}$, by (2.7) and (2.8) we have

$$
\Phi_{1 / 2}\left(\left[\varphi_{-1 / 2}\left(c_{1}\right), X\right]\right)=\left[\varphi_{-1 / 2}\left(c_{1}\right), X\right] .
$$

From the facts $\left[\varphi_{-1 / 2}\left(c_{1}\right), X\right] \in \mathfrak{g}_{1 / 2}$ and $\Phi_{1 / 2}\left(\mathfrak{g}_{1 / 2}\right) \subset \mathrm{g}_{1 / 2}^{(1)}$ it follows that $\left[\varphi_{-1 / 2}\left(c_{1}\right), X\right]$ belongs to $\mathfrak{g}_{1 / 2}^{(1)}$. We put $Y_{k}=\left[\partial / \partial z_{k}, X\right](1 \leq k \leq n)$. Then by $(2.8) \Phi_{0}\left(Y_{k}\right)$ $=Y_{k}$. From the fact $\left[g_{-1}, X\right] \subset g_{0}$ and Lemma 2.5 it follows that $Y_{k}$ is contained in $\mathfrak{g}_{0}^{(1)}$. By (2.8) we can see that

$$
\sigma\left(Y_{k}\right)=\left(\begin{array}{cc}
\sigma_{1}\left(Y_{k}\right) & 0 \\
0 & 0
\end{array}\right)
$$

Thus, $\operatorname{Im} \operatorname{Tr} \sigma_{1}\left(Y_{k}\right)=\operatorname{Im} \operatorname{Tr} \sigma\left(Y_{k}\right)=0$. Therefore by (1.10) we conclude 
that $X$ belongs to $\mathfrak{g}_{1}^{(1)}$. The assertion (3) was proved.

By (1) and (3) we have $[X, Y] \in \mathfrak{g}_{0}^{(1)}$ for $X \in \mathfrak{g}_{-1}, Y \in \mathfrak{g}_{1}$. Therefore we get $\Phi_{0}([X, Y])=\left[\Phi_{-1}(X), \Phi_{1}(Y)\right]$. Let

$$
\begin{aligned}
X= & 2 i \sum_{1 \leq k, l \leq n} F^{k}\left(w_{1}, c_{l}\right) z_{l} \partial / \partial z_{k}+\sum_{\substack{1 \leq l \leq n \\
1 \leq \alpha \leq m_{1}}} c_{l}^{\alpha} z_{l} \partial / \partial w_{\alpha} \\
& +\sum_{1 \leq \alpha, \beta, \gamma \leq m} b_{\beta \gamma}^{\alpha} w_{\beta} w_{\gamma} \partial / \partial w_{\alpha} \in g_{1 / 2} \quad\left(c_{l} \in W_{1}\right) .
\end{aligned}
$$

Then for each $d=d_{1}+d_{2} \in W=W_{1}+W_{2}$ we have

$$
\left[\Phi_{-1 / 2}\left(\varphi_{-1 / 2}(d)\right), \Phi_{1 / 2}(X)\right]=\left[\varphi_{-1 / 2}\left(d_{1}\right), \Phi_{1 / 2}(X)\right] \text {. }
$$

We can verify that $\rho\left(\left[\varphi_{-1 / 2}\left(d_{1}\right), \Phi_{1 / 2}(X)\right]=\left(4 \operatorname{Im} F^{k}\left(c_{l}, d_{1}\right)\right)\right.$ and the $(\alpha, \beta)$ component of the matrix $\sigma_{1}\left(\left[\varphi_{-1 / 2}\left(d_{1}\right), \Phi_{1 / 2}(X)\right]\right)$ is

$$
2 \sum_{\substack{1 \leq k \leq n \\ 1 \leq r \leq m_{1}}}\left(i F_{\beta r}^{k} \bar{d}^{r} c_{k}^{\alpha}+b_{\beta r}^{\alpha} d^{r}\right) \quad\left(1 \leq \alpha, \beta \leq m_{1}\right) .
$$

On the other hand, by the conditions $c_{l} \in W_{1}$ and $F\left(W_{1}, W_{2}\right)=(0)$ we have

$$
\begin{aligned}
{\left[\varphi_{-1 / 2}(d), X\right]=} & 4 \sum_{1 \leq k, l \leq n} \operatorname{Im} F^{k}\left(c_{l}, d_{1}\right) z_{l} \partial / \partial z_{k} \\
& +2 \sum_{1 \leq \alpha, \beta, \gamma \leq m}\left(i \sum_{1 \leq k \leq n} F_{\beta \gamma}^{k} \bar{d}^{r} c_{k}^{\alpha}+b_{\beta r}^{\alpha} d^{r}\right) w_{\beta} \partial / \partial w_{\alpha} .
\end{aligned}
$$

We can see that $b_{\beta \gamma}^{\alpha}=0$ if $1 \leq \alpha, \beta \leq m_{1}<\gamma \leq m$. In fact, by (2.3) and the condition $F\left(W_{1}, W_{2}\right)=(0)$ it follows that $\sum_{1 \leq \alpha \leq m_{1}} b_{\beta \gamma}^{\alpha} F_{\alpha \delta}^{k}=0\left(1 \leq \delta \leq m_{1}\right)$, which implies $F^{k}\left(\sum_{1 \leq \alpha \leq m_{1}} b_{\beta r}^{\alpha} f_{\alpha}, f_{\delta}\right)=0 \quad\left(1 \leq k \leq n, \quad 1 \leq \delta \leq m_{1}\right)$. So, $\sum_{1 \leq \alpha \leq m_{1}} b_{\beta \gamma}^{\alpha} f_{\alpha}=0$ and $b_{\beta \gamma}^{\alpha}=0 \quad\left(1 \leq \alpha, \beta \leq m_{1}<\gamma \leq m\right)$. Therefore by (2.5) we have

$$
\begin{aligned}
\Phi_{0}\left(\left[\varphi_{-1 / 2}(d), X\right]\right)= & 4 \sum_{1 \leq k, l \leq n} \operatorname{Im} F^{k}\left(c_{l}, d_{1}\right) z_{l} \partial / \partial z_{k} \\
& +2 \sum_{1 \leq \alpha, \beta, r \leq m_{1}}\left(i \sum_{1 \leq k \leq n} F_{\beta \gamma}^{k} \bar{d}^{r} c_{k}^{\alpha}+b_{\beta \gamma}^{\alpha} d^{r}\right) w_{\beta} \partial / \partial w_{\alpha},
\end{aligned}
$$

which implies that $\Phi_{0}\left(\left[\varphi_{-1 / 2}(d), X\right]\right)=\left[\Phi_{-1 / 2}\left(\varphi_{-1 / 2}(d)\right), \Phi_{1 / 2}(X)\right]$.

q.e.d.

By (2) in the above proposition we get

COROLLARY 2.7. If $\mathrm{g}_{1 / 2}^{(i)}=(0)(i=1,2)$, then $\mathrm{g}_{1 / 2}=(0)$.

2.3. Let $D(V, F)$ be a Siegel domain of type II in $R^{c} \times W$ : Let $D^{\prime}$ denote the associated tube domain with $D(V, F)$, i.e.,

$$
D^{\prime}=D(V, F) \cap\left(R^{c} \times\{0\}\right),
$$


which is isomorphic to the Siegel domain $D(V)$ of type I in $R^{c}$. It was proved by Kaup, Matsushima and Ochiai [6] that the subalgebra $g_{-1}+$ $\mathfrak{g}_{0}+\mathfrak{g}_{1}$ of $\mathfrak{g}_{h}$ is the Lie subalgebra corresponding to the subgroup of all automorphisms of $D(V, F)$ leaving the domain $D^{\prime}$ invariant. Let $\mathfrak{g}_{h}^{\prime}=\mathfrak{g}_{-1}^{\prime}$ $+\mathfrak{g}_{0}^{\prime}+\mathfrak{g}_{1}^{\prime}$ be the Lie algebra of all infinitesimal automorphisms of $D^{\prime}$. Then there exists a grade-preserving Lie algebra homomorphism $\xi$ of $\mathfrak{g}_{-1}$ $+\mathfrak{g}_{0}+\mathfrak{g}_{1}$ into $\mathfrak{g}_{h}^{\prime}=\mathfrak{g}_{-1}^{\prime}+\mathfrak{g}_{0}^{\prime}+\mathfrak{g}_{1}^{\prime}$;

$$
\xi: X \in \mathfrak{g}_{-1}+\mathfrak{g}_{0}+\mathfrak{g}_{1} \mapsto \xi(X) \in \mathfrak{g}_{h}^{\prime},
$$

where $\xi(X)$ is the vector field which is the restriction of $X$ to $D^{\prime}$.

As a corollary to Proposition 2.6 we have the following proposition which will be used in order to determine the subspace $g_{1}$ of $g_{h}$.

Proposition 2.8. If $\mathfrak{g}_{1 / 2}=(0)$, then $\mathfrak{g}_{1}$ is a subspace of $\mathfrak{g}_{1}^{\prime}$ and the map $\xi$ restricted to $\mathfrak{g}_{1}$ is an identity.

Proof. We put $W_{1}=(0)$ and $W_{2}=W$. Then the Siegel domains $D\left(V, F_{1}\right)$ and $D\left(V, F_{2}\right)$ coincide with $D^{\prime}$ and $D(V, F)$, respectively. Therefore $\mathfrak{g}_{h}^{(1)}=g_{h}^{\prime}$ and $g_{h}^{(2)}=g_{h}$. It is easy to see that the map $\Phi$ restricted to $\mathfrak{g}_{-1}+\mathfrak{g}_{0}+\mathfrak{g}_{1}$ coincides with the map $\xi$ (cf. (2.5)). Thus our assertions follow from (3) of Proposition 2.6.

q.e.d.

\section{§ 3. Automorphisms of Siegel domains over self-dual cones}

In this section we calculate infinitesimal automorphisms of the homogeneous Siegel domains over self-dual cones (except circular cones) which were constructed by Pjateckii-Sapiro [10].

3.1. We will use the following notations and well-known results for irreducible self-dual cones.

1) The cone $H^{+}(p, R)$.

Let $R=H(p, \boldsymbol{R})$ be the real vector space of all real symmetric matrices of degree $p$. We denote by $H^{+}(p, R)$ the cone of all positive definite matrices in $R$. Then $\operatorname{dim} R=\frac{1}{2} p(p+1)$. Let $E_{i j}$ denote a square matrix of degree $p$ whose $(i, j)$-component is one and others are zero. We define a base $\left\{e_{i j}\right\}_{1 \leq i \leq j \leq p}$ of $R$ by $e_{i i}=E_{i i}(1 \leq i \leq p)$ and $e_{i j}=E_{i j}$ $+E_{j i}(1 \leq i<j \leq p) . \quad\left(z_{i j}\right)_{1 \leq i \leq j \leq p}$ denotes the coordinate system of $R^{c}$ associated with the base $\left\{e_{i j}\right\}_{1 \leq i \leq j \leq p}$.

It is known in [17] that the Lie algebra $g\left(H^{+}(p, R)\right)$ consists of all linear endomorphisms $\tilde{A}$ of the form; 


$$
\tilde{A}: X \in R \mapsto A X+X^{t} A \in R,
$$

where $A$ is an element of $\operatorname{gr}(p, R)$.

2) The cone $H^{+}(p, C)$.

Let $R=H(p, C)$ be the real vector space of all hermitian matrices of degree $p$. We denote by $H^{+}(p, C)$ the cone of all positive definite matrices in $R$. Then $\operatorname{dim} R=p^{2}$. We define a base $\left\{e_{i i}(1 \leq i \leq p), e_{i j, s}\right.$ $(1 \leq i<j \leq p, s=1,2)\}$ of $R$ by $e_{i i}=E_{i i}(1 \leq i \leq p), e_{i j, 1}=E_{i j}+E_{j i}$ and $e_{i j, 2}=i\left(E_{i j}-E_{j i}\right) \quad(1 \leq i<j \leq p) . \quad\left(z_{i i}(1 \leq i \leq p), z_{i j, s} \quad(1 \leq i<j\right.$ $\leq p, s=1,2)$ ) denotes the coordinate system of $R^{c}$ associated with the base $\left\{e_{i i}, e_{i j, s}\right\}$.

It is known in [17] that the Lie algebra $\mathrm{g}\left(\mathrm{H}^{+}(p, C)\right)$ consists of all linear endomorphisms $\tilde{A}$ of the form;

$$
\tilde{A}: X \in R \mapsto A X+X^{t} \bar{A} \in R,
$$

where $A$ is an element of $\mathfrak{g l}(p, C)$.

3) The cone $H^{+}(p, K)$.

Let $R=H(p, K)$ be the real vector space of all hermitian matrices $X$ of degree $2 p$ satisfying the condition; $X J=J \bar{X}$, where

$$
J=\left(\begin{array}{ccc}
j & & 0 \\
& \ddots & \\
0 & & j
\end{array}\right) \text { and } j=\left(\begin{array}{cc}
0 & 1 \\
-1 & 0
\end{array}\right) .
$$

We denote by $H^{+}(p, K)$ the cone of all positive definite matrices in $R$. Let $X=\left(X_{k l}\right)$ be a hermitian matrix of degree $2 p$, where $X_{k l}$ is a $2 \times 2$ minor matrix of $X(1 \leq k, l \leq p)$. Then $X$ belongs to $R$ if and only if $X_{k l}$ is represented as follows;

$$
X_{k k}=\left(\begin{array}{cc}
x_{k k} & 0 \\
0 & x_{k k}
\end{array}\right)(1 \leq k \leq p), \quad X_{k l}=\left(\begin{array}{cc}
x_{k l} & y_{k l} \\
-\bar{y}_{k l} & \bar{x}_{k l}
\end{array}\right)(1 \leq k<l \leq p),
$$

where $x_{k k} \in R$ and $x_{k l}, y_{k l} \in C$. Thus we have $\operatorname{dim} R=p(2 p-1)$. We define a base $\left\{e_{i i}(1 \leq i \leq p), e_{i j, s}(1 \leq i<j \leq p, 1 \leq s \leq 4)\right\}$ of $R$ by $e_{i i}=E_{2 i-12 i-1}+E_{2 i 2 i}(1 \leq i \leq p), e_{i j, 1}=E_{2 i-12 j-1}+E_{2 i 2 j}, e_{i j, 2}=i\left(E_{2 i-12 j-1}\right.$ $\left.-E_{2 i 2 j}\right), e_{i j, 3}=E_{2 i-12 j}-E_{2 i 2 j-1}, e_{i j, 4}=i\left(E_{2 i-12 j}+E_{2 i 2 j-1}\right)(1 \leq i<j \leq p)$, where $E_{i j}$ is the square matrix of degree $2 p$ whose $(i, j)$-component is one and others are zero. $\left(z_{i i}(1 \leq i \leq p), z_{i j, s}(1 \leq i<j \leq p, 1 \leq s \leq 4)\right)$ denotes the coordinate system of $R^{c}$ associated with the base $\left\{e_{i i}, e_{i j, s}\right\}$.

It is known in [17] that the Lie algebra $\mathrm{g}\left(\mathrm{H}^{+}(p, K)\right)$ consists of all 
linear endomorphisms $\tilde{A}$ of the form;

$$
\tilde{A}: X \in R \mapsto A X+X^{t} \bar{A} \in R,
$$

where $A$ is an element of $\operatorname{gl}(2 p, C)$ satisfying the condition $A J=J \bar{A}$.

3.2. As an application of Theorem 2.1 we have

LEMMA 3.1. For each of the homogeneous Siegel domains $D(V, F)$ given in the following (1),(2) and (3), the Lie algebra $\mathfrak{g}_{h}$ coincides with the subalgebra $\mathfrak{g}_{a}$.

(1) $V=H^{+}(p, \boldsymbol{R}), W=M(p, q ; C)(p \geq 2)$,

$$
F(u, v)=\frac{1}{2}\left(u^{t} \bar{v}+\bar{v}^{t} u\right) \quad \text { for } u, v \in W .
$$

(2) $V=H^{+}(p, C), W=M\left(p, q_{1} ; C\right)+M\left(p, q_{2} ; C\right)$ (direct sum, $\left.p \geq 2\right)$,

$$
\begin{aligned}
F(u, v)=\frac{1}{2}\left(u^{(1)} t \bar{v}^{(1)}+\bar{v}^{(2) t} u^{(2)}\right) \\
\text { for } u=u^{(1)}+u^{(2)}, v=v^{(1)}+v^{(2)} \in W .
\end{aligned}
$$

(3) $V=H^{+}(p, K), W=M(2 p, q ; C)(p, q \geq 2)$,

$$
F(u, v)=\frac{1}{2}\left(u^{t} \bar{v}+J \bar{v}^{t} u^{t} J\right) \quad \text { for } u, v \in W .
$$

Proof. First we show that for each Siegel domain $D(V, F)$ in (1), (2) and (3), the subalgebra $\rho\left(\mathfrak{g}_{0}\right)$ of $g(V)$ coincides with $g(V)$.

Case (1): For each $\tilde{A} \in \mathfrak{g}(V)(A \in \mathfrak{g l}(p, R))$ we define a complex linear endomorphism $B$ of $W$ by

$$
B: u \in W \mapsto A u \in W,
$$

where $A u$ means a usual matrix multiplication of $A$ and $u$. Then by (3.1) we have

$$
\tilde{A} F(u, u)=F(B u, u)+F(u, B u)
$$

for every $u \in W$. Hence by (1.4) $\tilde{A}$ is contained in $\rho\left(g_{0}\right)$. Therefore we have $\rho\left(\mathfrak{g}_{0}\right)=\mathfrak{g}(V)$.

Case (2): For each $\tilde{A} \in \mathrm{g}(V)(A \in \mathrm{gl}(p, C))$ we define a complex linear endomorphism $B$ of $W$ by

$$
B: u=u^{(1)}+u^{(2)} \in W \mapsto A u^{(1)}+\bar{A} u^{(2)} \in W .
$$

Then by using (3.2) we can verify

$$
\tilde{A} F(u, u)=F(B u, u)+F(u, B u)
$$


for every $u \in W$. It follows from (1.4) that $\tilde{A}$ belongs to $\rho\left(g_{0}\right)$. Thus, we have $\rho\left(g_{0}\right)=g(V)$.

Case (3): For each $\tilde{A} \in \mathfrak{g}(V) \quad(A \in \mathfrak{g l}(2 p, C), A J=J \bar{A})$ we define a complex linear endomorphism $B$ of $W$ by

$$
B: u \in W \mapsto A u \in W .
$$

Then by (3.3) we have

$$
\tilde{A} F(u, u)=F(B u, u)+F(u, B u)
$$

for every $u \in W$. Hence by (1.4) $\tilde{A}$ belongs to $\rho\left(g_{0}\right)$ and $\rho\left(g_{0}\right)=g(V)$.

Each cone $V$ in (1), (2) and (3) is an irreducible homogeneous selfdual cone. On the other hand, it was proved by Rothaus [11] that for an irreducible homogeneous self-dual cone $V$, the Lie algebra $g(V)$ is irreducible. Therefore the representation $\rho$ is irreducible. Furthermore each domain $D(V, F)$ in (1), (2) and (3) is non-symmetric (cf. [10]). Thus, from Theorem 2.1 we conclude that $g_{h}=g_{a}$.

Now we consider degenerate Siegel domains over the cones $V=$ $H^{+}(p, \boldsymbol{F}) \quad(p \geq 2)$, where $\boldsymbol{F}$ is $\boldsymbol{R}$ or $\boldsymbol{C}$ or $\boldsymbol{K}$. Let $\boldsymbol{F}$ be a $V$-hermitian form on a complex vector space $W$ of dimension $m(m>0)$. Then we get

LEMMA 3.2. If there exists a positive integer $q(q<p)$ such that the linear closure of the set $\{F(u, u) ; u \in W\}$ in $R$ coincides with the proper subspace $\left(\begin{array}{cc}H(q, F) & 0 \\ 0 & 0\end{array}\right)$ of $R$, then $g_{1 / 2}=(0)$.

Proof. Case $\boldsymbol{F}=\boldsymbol{R}$ : We show that if a linear endomorphism $\tilde{A} \epsilon$ $\mathfrak{g}(V)$ belongs to $\rho\left(\mathrm{g}_{0}\right)$, then $A$ must be of the form;

$$
A=\left(\begin{array}{ll}
a & b \\
0 & c
\end{array}\right)
$$

where $a \in \operatorname{gl}(q, \boldsymbol{R}), \quad b \in M(q, p-q ; \boldsymbol{R})$ and $c \in \operatorname{gl}(p-q, \boldsymbol{R})$. In fact, let $\tilde{A} \in \rho\left(\mathfrak{g}_{0}\right), A=\left(\begin{array}{ll}a & b \\ d & c\end{array}\right)$. Then by (1.4) there exists $B \in \mathfrak{g l}(W)$ such that $(\tilde{A}, B)$ satisfies the condition; $\tilde{A} F(u, u)=F(B u, u)+F(u, B u)$ for every $u \in W$. Therefore $A$ must satisfy the following; for each $Y \in H(q, \boldsymbol{R})$,

$$
A\left(\begin{array}{ll}
Y & 0 \\
0 & 0
\end{array}\right)+\left(\begin{array}{ll}
Y & 0 \\
0 & 0
\end{array}\right)^{t} A \text { belongs to }\left(\begin{array}{cc}
H(q, R) & 0 \\
0 & 0
\end{array}\right)
$$


which implies $d=0$.

Now we want to show $g_{1 / 2}=0$. For each $X \in g_{1 / 2}$, by (2.2) and (2.4) there exist $c_{k l} \in W(1 \leq k \leq l \leq p)$ such that

$$
\frac{1}{4} \rho\left(\left[\varphi_{-1 / 2}(d), X\right]\right)=\left(\operatorname{Im} F^{i j}\left(c_{k l}, d\right)\right)
$$

for every $d \in W$. From our assumption we can see that $F^{i j}=0$ if $j>q$. Therefore, the linear endomorphism $\rho\left(\left[\varphi_{-1 / 2}(d), X\right]\right)$ maps the space $R=$ $H(p, \boldsymbol{R})$ into the proper subspace $\left(\begin{array}{cc}H(q, \boldsymbol{R}) & 0 \\ 0 & 0\end{array}\right)$ of $R$. On the other hand, from (3.4) there exists $A \in \operatorname{gr}(p, R)$ of the form: $A=\left(\begin{array}{ll}a & b \\ 0 & c\end{array}\right)$ satisfying $\rho\left(\left[\varphi_{-1 / 2}(d), X\right]\right)=\tilde{A}$. Thus, for each $Y_{1} \in H(q, \boldsymbol{R}), Y_{2} \in M(q, p-q ; \boldsymbol{R})$ and $Y_{3} \in H(p-q, \boldsymbol{R})$,

$$
\left(\begin{array}{ll}
a & b \\
0 & c
\end{array}\right)\left(\begin{array}{ll}
Y_{1} & Y_{2} \\
{ }^{t} Y_{2} & Y_{3}
\end{array}\right)+\left(\begin{array}{cc}
Y_{1} & Y_{2} \\
{ }^{t} Y_{2} & Y_{3}
\end{array}\right)\left(\begin{array}{cc}
{ }^{t} a & 0 \\
{ }^{t} b & { }^{t} c
\end{array}\right) \text { belongs to }\left(\begin{array}{cc}
H(q, R) & 0 \\
0 & 0
\end{array}\right) \text {. }
$$

Hence we get $a Y_{2}+Y_{2}^{t} c+b Y_{3}=0$ and $c Y_{3}+Y_{3}^{t} c=0$, which implies $b=0$. We can see that $a=0$ and $c=0$ by taking $Y_{2}$ and $Y_{3}$ suitably. So, $\tilde{A}=0$ and $\rho\left(\left[\varphi_{-1 / 2}(d), X\right]\right)=0$. By Proposition 2.3 we conclude that $\mathfrak{g}_{1 / 2}=(0)$.

Case $F=C$ : We proceed analogously as in the above case. Let $\tilde{A} \in \mathfrak{g}(V)$ belong to $\rho\left(g_{0}\right)$. Then by (1.4) it can be easily verified that $A$ must be of the form;

$$
A=\left(\begin{array}{ll}
a & b \\
0 & c
\end{array}\right)
$$

where $a \in \mathfrak{g l}(q, C), b \in M(q, p-q ; C)$ and $c \in \mathfrak{g l}(p-q, C)$.

Now we show $\mathfrak{g}_{1 / 2}=(0)$. Let $X \in g_{1 / 2}$. Then by (2.2) and (2.4) there exist $c_{k k}(1 \leq k \leq p), c_{k l, t}(1 \leq k<l \leq p, t=1,2) \in W$ such that

$$
\frac{1}{4} \rho\left(\left[\varphi_{-1 / 2}(d), X\right]\right)=\left(\operatorname{Im} F^{i j, s}\left(c_{k l, t}, d\right)\right)
$$

for each $d \in W$, where we put $F^{i i, s}=F^{i i}, c_{i i, s}=c_{i i}$ and $F(u, v)=$ $\sum F^{i j, s}(u, v) e_{i j, s}$. From our assumption it follows that $F^{i j, s}=0$ if $j>q$. Therefore the linear endomorphism $\rho\left(\left[\varphi_{-1 / 2}(d), X\right]\right)$ maps the space $R=$ $H(p, C)$ into the proper subspace $\left(\begin{array}{cc}H(q, C) & 0 \\ 0 & 0\end{array}\right)$ of $R$. On the other hand, there exists $A \in \mathfrak{g l}(p, C)$ of the form (3.5) such that $\rho\left(\left[\varphi_{-1 / 2}(d), X\right]\right)=\tilde{A}$. Thus for each $Y_{1} \in H(q, C), Y_{2} \in M(q, p-q ; C)$ and $Y_{3} \in H(p-q, C)$, 


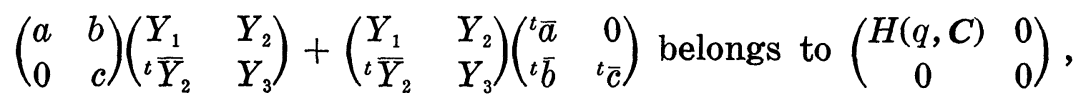

that is, $a Y_{2}+Y_{2}^{t} \bar{c}+b Y_{3}=0$ and $c Y_{3}+Y_{3}^{t} \bar{c}=0$. Taking $Y_{2}$ and $Y_{3}$ suitably we have $b=0, a=i \theta E_{q}$ and $c=i \theta E_{p-q}$, where $\theta$ is a real number. By considering (3.2) we get $\tilde{A}=0$. Therefore $\rho\left(\left[\varphi_{-1 / 2}(d), X\right]\right)$ $=0$ for every $d \in W$. So, by Proposition 2.3, $\mathrm{g}_{1 / 2}=(0)$.

Case $F=K$ : By the same considerations as in the above, we can see that if $\tilde{A} \in \mathfrak{g}(V)$ belongs to $\rho\left(\mathfrak{g}_{0}\right)$, then $A$ must be of the form;

$$
A=\left(\begin{array}{ll}
a & b \\
0 & c
\end{array}\right)
$$

where $a \in \mathfrak{g}(2 q, C), b \in M(2 q, 2(p-q) ; C)$ and $c \in \mathfrak{g l}(2(p-q), C)$ satisfying $a J_{1}=J_{1} \bar{a}, c J_{2}=J_{2} \bar{c}, b J_{2}=J_{1} \bar{b}, J=\left(\begin{array}{cc}J_{1} & 0 \\ 0 & J_{2}\end{array}\right)$ (cf. (3.3)).

Now we want to show $g_{1 / 2}=(0)$. For each $X \in g_{1 / 2}$, by (2.2) and (2.4) there exist $c_{k k}(1 \leq k \leq p), c_{k l, t}(1 \leq k<l \leq p, 1 \leq t \leq 4) \in W$ such that

$$
\frac{1}{4} \rho\left(\left[\varphi_{-1 / 2}(d), X\right]\right)=\left(\operatorname{Im} F^{i j, s}\left(c_{k l, t}, d\right)\right)
$$

for every $d \in W$, where we put $F^{i i, s}=F^{i i}, c_{i i, s}=c_{i i}$ and $F(u, v)=$ $\sum F^{i j, s}(u, v) e_{i j, s} . \quad$ By our assumption, $F^{i j, s}=0$ if $j>q$. Therefore the linear endomorphism $\rho\left(\left[\varphi_{-1 / 2}(d), X\right]\right)$ maps the space $R=H(p, K)$ into the proper subspace $\left(\begin{array}{cc}H(q, K) & 0 \\ 0 & 0\end{array}\right)$ of $R$. On the other hand, there exists $\tilde{A} \in \rho\left(\mathrm{g}_{0}\right)$ of the form (3.6) such that $\rho\left(\left[\varphi_{-1 / 2}(d), X\right]\right)=\tilde{A}$. Thus, for each $Y_{1} \in H(q, K), Y_{2} \in M(2 q, 2(p-q) ; C)$ and $Y_{3} \in H(p-q, K)$ satisfying $Y_{2} J_{2}$ $=J_{1} \bar{Y}_{2}$,

$$
\left(\begin{array}{ll}
a & b \\
0 & c
\end{array}\right)\left(\begin{array}{cc}
Y_{1} & Y_{2} \\
t \bar{Y}_{2} & Y_{3}
\end{array}\right)+\left(\begin{array}{cc}
Y_{1} & Y_{2} \\
{ }^{t} \bar{Y}_{2} & Y_{3}
\end{array}\right)\left(\begin{array}{cc}
{ }^{t} \bar{a} & 0 \\
t \bar{b} & { }^{t} \bar{c}
\end{array}\right) \text { belongs to }\left(\begin{array}{cc}
H(q, K) & 0 \\
0 & 0
\end{array}\right) \text {. }
$$

Hence we have

$$
a Y_{2}+Y_{2}^{t} \bar{c}+b Y_{3}=0 \text { and } c Y_{3}+Y_{3}^{t} \bar{c}=0 .
$$

Taking $Y_{2}$ and $Y_{3}$ suitably we get $a=0, b=0$ and $c=0$. So, $\tilde{A}=0$ and $\rho\left(\left[\mathfrak{g}_{-1 / 2}, X\right]\right)=(0)$. From Proposition 2.3 it follows that $g_{1 / 2}=(0)$.

q.e.d.

3.3. In this paragraph we calculate infinitesimal automorphisms of all homogeneous Siegel domains of type II over the cone $V=H^{+}(p, \boldsymbol{R})$ $(p \geq 2)$. 
Let $s$ be a positive integer and $r(t)$ be a non-decreasing integer valued function defined on an interval $[1, s]$ such that $1 \leq r(1), r(s) \leq p$. Let $\mathrm{W}$ be the complex vector space of all complex $p \times s$-matrices $u=$ $\left(u_{i j}\right)$ such that $u_{i j}=0$ if $i>r(j)$. We put $F(u, v)=\frac{1}{2}\left(u^{t} \bar{v}+\bar{v}^{t} u\right)$ for $u, v \in W$. Then it is known in [10] that $F$ is a $V$-hermitian form on $W$ and the Siegel domain $D(V, F)$ is homogeneous. We note that every homogeneous Siegel domain of type II over the cone $H^{+}(p, \boldsymbol{R})(p \geq 2)$ is isomorphic to the one given here (cf. [10], [13]). It was proved by Kaneyuki and Sudo [4] that the Siegel domain $D(V, F)$ is non-degenerate if and only if $r(s)=p$.

THEOREM 3.3." For a Siegel domain $D(V, F)$ mentioned above, the subspaces $\mathrm{g}_{1 / 2}$ and $\mathrm{g}_{1}$ of $\mathrm{g}_{h}$ are given as follows;

$\mathrm{g}_{1 / 2}=(0)$,

$\mathrm{g}_{1}$ is isomorphic to the vector space $H(p-r(s), \boldsymbol{R})$.

Proof. First we suppose that $D(V, F)$ is degenerate. Then $r(s)<p$ and the linear closure of the set $\{F(u, u) ; u \in W\}$ in $R$ coincides with the proper subspace $\left(\begin{array}{cc}H(q, \boldsymbol{R}) & 0 \\ 0 & 0\end{array}\right)$ of $R$, where $q=r(s)$ (cf. [4]). Hence, by Lemma 3.2 we have $g_{1 / 2}=(0)$.

Now we determine $g_{1}{ }^{2}$ ) We consider the associated tube domain $D^{\prime}$ with $D(V, F)$ (cf. (2.9)). It is known in [10] that $D^{\prime}$ is the classical domain of type (III) and the Lie algebra $g_{h}^{\prime}=\mathfrak{g}_{-1}^{\prime}+\mathfrak{g}_{0}^{\prime}+\mathfrak{g}_{1}^{\prime}$ of all infinitesimal automorphisms of $D^{\prime}$ can be identified with $\mathfrak{S p}(p, R)$ as follows (cf. [10], Chap. 2, §7);

$$
\begin{aligned}
\mathfrak{g}_{h}^{\prime}=\mathfrak{S p}(p, \boldsymbol{R}) & =\left\{\left(\begin{array}{rr}
A & \boldsymbol{B} \\
C & -{ }^{t} A
\end{array}\right) ; A \in \mathfrak{g l}(p, \boldsymbol{R}), B, C \in H(p, \boldsymbol{R})\right\}, \\
\mathfrak{g}_{-1}^{\prime} & =\left(\begin{array}{cc}
0 & H(p, \boldsymbol{R}) \\
0 & 0
\end{array}\right), \quad \mathrm{g}_{1}^{\prime}=\left(\begin{array}{cc}
0 & 0 \\
H(p, \boldsymbol{R}) & 0
\end{array}\right), \\
\mathfrak{g}_{0}^{\prime} & =\left\{\left(\begin{array}{rr}
A & 0 \\
0 & -{ }^{t} A
\end{array}\right) ; A \in \mathfrak{g l}(p, \boldsymbol{R})\right\} .
\end{aligned}
$$

For each $g=\left(\begin{array}{ll}E_{p} & 0 \\ C & E_{p}\end{array}\right) \in \exp g_{1}^{\prime}, g$ acts on $D^{\prime}$ by

1) If $s=1$, then this theorem was proved by Tanaka [14] and Murakami [8]. Nakajima [18] calculated the dimensions of $g_{1 / 2}$ and $g_{1}$ of this theorem by using different methods.

2) This idea of determining $g_{1}$ is due to Murakami [8]. 


$$
g: z \in D^{\prime} \mapsto z\left(C z+E_{p}\right)^{-1} \in D^{\prime} .
$$

The image $\xi\left(g_{0}\right)$ of $g_{0}$ is given by

$$
\xi\left(g_{0}\right)=\left\{\left(\begin{array}{rr}
A & 0 \\
0 & -{ }^{t} A
\end{array}\right) \in \mathfrak{g}_{0}^{\prime} ; \tilde{A} \in \rho\left(g_{0}\right)\right\} \quad \text { (cf. (2.10)) . }
$$

We want to show that $\xi\left(\mathfrak{g}_{1}\right)$ coincides with the following subspace of $\mathfrak{g}_{1}^{\prime}$;

$$
\left\{\left(\begin{array}{ll}
0 & 0 \\
Y & 0
\end{array}\right) \in g_{1}^{\prime} ; Y=\left(\begin{array}{ll}
0 & 0 \\
0 & y
\end{array}\right), y \in H(p-q, \boldsymbol{R})\right\}
$$

Let $X \in \mathfrak{g}_{1}$. Then, since $\xi(X) \in \mathfrak{g}_{1}^{\prime}$, there exists $Y \in H(p, R)$ such that $\xi(X)=\left(\begin{array}{ll}0 & 0 \\ Y & 0\end{array}\right)$. By the conditions $\xi\left(g_{-1}\right)=\mathfrak{g}_{-1}^{\prime}$ and $\left[g_{-1}, X\right] \subset g_{0}$ we have $\left[g_{-1}^{\prime}, \xi(X)\right] \subset \xi\left(g_{0}\right)$. Therefore, for each $B \in H(p, R), \widetilde{B Y}$ belongs to $\rho\left(g_{0}\right)$. So, $B Y$ must be of the form (3.4) for each $B \in H(p, R)$, which implies that $Y$ must be of the form (3.7). Conversely let $Y$ be an element in $H(p, R)$ of the form (3.7). We define the map $g_{t}(t \in R)$ of $D(V, F)$ into $R^{c} \times W$ by

$$
g_{t}:(z, u) \in D(V, F) \mapsto\left(z\left(t Y z+E_{p}\right)^{-1}, u\right) \in R^{c} \times W .
$$

Then we can easily verify (cf. [8]) that

$$
\operatorname{Im}\left(z\left(t Y z+E_{p}\right)^{-1}\right)=\overline{{ }^{t}\left(t Y z+E_{p}\right)^{-1}} \operatorname{Im} z\left(t Y z+E_{p}\right)^{-1}
$$

and

$$
{ }^{t}\left(\overline{t Y z+E_{p}}\right)^{-1} F(u, u)\left(t Y z+E_{p}\right)^{-1}=F(u, u)
$$

for each $u \in W$.

Thus, $g_{t}$ is a one-parameter group of transformations of $D(V, F)$ and $g_{t}$ induces a vector field $X \in \mathfrak{g}_{1}$ such that $\xi(X)=\left(\begin{array}{ll}0 & 0 \\ Y & 0\end{array}\right)$. By the fact $\mathfrak{g}_{1 / 2}$ $=(0)$ and Proposition 2.8 we conclude that $g_{1}$ is isomorphic to the vector space $H(p-q, \boldsymbol{R})$.

Now we suppose that $D(V, F)$ is non-degenerate. If $r(1)=p$, then $W$ coincides with $M(p, s ; C)$ and the Siegel domain $D(V, F)$ is the one given in (1) of Lemma 3.1. So, we can assume that $s \geq 2$ and $r(1)<p$. We put $t_{0}=\min \{t \in[1, s] ; t$ is an integer such that $r(t)=p\}$ and define the complex subspaces $W_{i}(i=1,2)$ of $W$ by

$$
W_{1}=\left\{u=\left(u_{i j}\right) \in W ; u_{i j}=0 \text { if } j<t_{0}\right\}
$$

and 


$$
W_{2}=\left\{u=\left(u_{i j}\right) \in W ; u_{i j}=0 \text { if } j \geq t_{0}\right\} .
$$

Then it can be seen that

$$
W=W_{1}+W_{2}\left(\text { direct sum) and } F\left(W_{1}, W_{2}\right)=(0)\right. \text {. }
$$

We denote by $F_{i}$ the restriction of $F$ to the subspace $W_{i}$. Then the vector space $W_{1}$ is isomorphic to $M\left(p, s-t_{0}+1 ; C\right)$, and the Siegel domain $D\left(V, F_{1}\right)$ in $R^{c} \times W_{1}$ is isomorphic to the one given in (1) of Lemma 3.1. Thus $\mathfrak{g}_{1 / 2}^{(1)}=(0)$.

On the other hand, for the Siegel domain $D\left(V, F_{2}\right)$ in $R^{c} \times W_{2}$ we can see that the linear closure of the set $\left\{F_{2}(u, u) ; u \in W_{2}\right\}$ in $R$ coincides with the proper subspace $\left(\begin{array}{cc}H(q, R) & 0 \\ 0 & 0\end{array}\right)$ of $R$, where $q=r\left(t_{0}-1\right)$. Hence by Lemma 3.2 we have $\mathfrak{g}_{1 / 2}^{(2)}=(0)$. From Corollary 2.7 it follows that $\mathfrak{g}_{1 / 2}$ $=(0)$. Therefore by Proposition 2.2 we get $g_{h}=g_{a}$.

q.e.d.

3.4. In this paragraph we consider the Siegel domains of type II over the cone $V=H^{+}(p, C)(p \geq 2)$.

Let $s_{1}$ and $s_{2}$ be two positive integers. Let $r_{i}(t)$ be a non-decreasing integer valued function defined on an interval $\left[1, s_{i}\right]$ such that $0 \leq r_{i}(t)$ and $r_{i}(t) \leq p(i=1,2)$. We denote by $W^{(i)}$ the complex vector space of all complex $p \times s_{i}$-matrices $u^{(i)}=\left(u_{k l}^{(i)}\right)$ such that $u_{k l}^{(i)}=0$ if $k>r_{i}(l)$. Let $W$ be the direct sum of the vector spaces $W^{(1)}$ and $W^{(2)}$. We put $F(u, v)=\frac{1}{2}\left(u^{(1) t} \bar{v}^{(1)}+\bar{v}^{(2) t} u^{(2)}\right)$ for $u=u^{(1)}+u^{(2)}, v=v^{(1)}+v^{(2)} \in W=W^{(1)}$ $+W^{(2)}$. Then it is known in [10] that the map $F$ is a $V$-hermitian form on $W$ and the Siegel domain $D\left(V, F^{\prime}\right)$ is homogeneous. Furthermore it was proved in [4] that the Siegel domain $D(V, F)$ is non-degenerate if and only if $r_{1}\left(s_{1}\right)=p$ or $r_{2}\left(s_{2}\right)=p$.

THEOREM 3.4.3) (i) If a Siegel domain $D(V, F)$ mentioned above is degenerate, then the subspaces $\mathfrak{g}_{1 / 2}$ and $\mathfrak{g}_{1}$ of $\mathfrak{g}_{h}$ are given by

$\mathfrak{g}_{1 / 2}=(0)$,

$\mathrm{g}_{1}$ is isomorphic to the vector space $H(p-q, C)$, where $q=\max \left(r_{1}\left(s_{1}\right), r_{2}\left(s_{2}\right)\right)$.

(ii) If $r_{1}\left(s_{1}\right)=r_{2}\left(s_{2}\right)=p$, then $\mathfrak{g}_{h}=\mathfrak{g}_{a}$.

Proof. First we consider the case (i). The linear closure of the

3) Nakajima [18] calculated the dimensions of $g_{1 / 2}$ and $g_{1}$ of this theorem by using different methods. 
set $\{F(u, u) ; u \in W\}$ in $R$ coincides with the proper subspace $\left(\begin{array}{cc}H(q, C) & 0 \\ 0 & 0\end{array}\right)$ of $R$ (cf. [4]). Thus, by Lemma 3.2 it follows $\mathfrak{g}_{1 / 2}=(0)$.

Now we determine $\mathfrak{g}_{1}$. We consider the tube domain $D^{\prime}$ associated with $D(V, F)$ (cf. (2.9)). Then it is known in [10] that $D^{\prime}$ is the classical domain of type (I). The Lie algebra $\mathfrak{g}_{h}^{\prime}=\mathfrak{g}_{-1}^{\prime}+\mathfrak{g}_{0}^{\prime}+\mathfrak{g}_{1}^{\prime}$ of all infinitesimal automorphisms of $D^{\prime}$ can be identified with $\mathfrak{g} u(p, p)$ as follows (cf. [10], Chap. 2, §6);

$$
\begin{aligned}
\mathfrak{g}_{h}^{\prime} & =\mathfrak{g} \mathfrak{u}(p, p) \\
& =\left\{\left(\begin{array}{cc}
A & B \\
C & -{ }^{t} \bar{A}
\end{array}\right) ; A \in \mathfrak{g l}(p, C), B, C \in H(p, C)\right\} \quad\left(\bmod \left\{i \theta E_{2 p} ; \theta \in R\right\}\right), \\
\mathfrak{g}_{-1}^{\prime} & =\left(\begin{array}{cc}
0 & H(p, C) \\
0 & 0
\end{array}\right), \quad \mathfrak{g}_{1}^{\prime}=\left(\begin{array}{cc}
0 & 0 \\
H(p, C) & 0
\end{array}\right), \\
\mathfrak{g}_{0}^{\prime} & =\left\{\left(\begin{array}{cc}
A & 0 \\
0 & -{ }^{t} \frac{0}{A}
\end{array}\right) ; A \in \operatorname{gl}(p, C)\right\} \quad\left(\bmod \left\{i \theta E_{2 p} ; \theta \in R\right\}\right) .
\end{aligned}
$$

Each $g=\left(\begin{array}{ll}E_{p} & 0 \\ C & E_{p}\end{array}\right)\left(\in \exp g_{1}^{\prime}\right)$ acts on $D^{\prime}$ by

$$
g: z \in D^{\prime} \mapsto z\left(C z+E_{p}\right)^{-1} \in D^{\prime} .
$$

The image $\xi\left(\mathfrak{g}_{0}\right)$ of $\mathfrak{g}_{0}$ is the subalgebra of $\mathfrak{g}_{0}^{\prime}$ given by

$$
\xi\left(g_{0}\right)=\left\{\left(\begin{array}{lr}
A & 0 \\
0 & -{ }^{t} \frac{A}{A}
\end{array}\right) \in \mathfrak{g}_{0}^{\prime} ; \tilde{A} \in \rho\left(\mathfrak{g}_{0}\right)\right\} .
$$

We want to show that the subspace $\xi\left(\mathfrak{g}_{1}\right)$ of $\mathfrak{g}_{1}^{\prime}$ coincides with the following subspace of $\mathfrak{g}_{1}^{\prime}$;

$$
\left\{\left(\begin{array}{ll}
0 & 0 \\
Y & 0
\end{array}\right) ; Y=\left(\begin{array}{ll}
0 & 0 \\
0 & y
\end{array}\right), y \in H(p-q, C)\right\} .
$$

In fact, let $X \in \mathfrak{g}_{1}$. Then $\xi(X)$ belongs to $\mathfrak{g}_{1}^{\prime}$ and $\xi(X)$ is represented as

$$
\xi(X)=\left(\begin{array}{ll}
0 & 0 \\
Y & 0
\end{array}\right), \quad Y \in H(p, C) .
$$

From the condition $\left[\mathfrak{g}_{-1}, X\right] \subset \mathfrak{g}_{0}$ and the fact $\xi\left(\mathfrak{g}_{-1}\right)=\mathfrak{g}_{-1}^{\prime}$, we have $\left[g_{-1}^{\prime}, \xi(X)\right] \subset \xi\left(g_{0}\right)$. Thus it can be seen that, for each $B \in H(p, C), B Y$ must be of the form (3.5). It follows that $Y$ must be of the form (3.8).

Conversely let $Y$ be an element in $H(p, C)$ of the form (3.8). We define the map $g_{t}(t \in R)$ of $D(V, F)$ into $R^{c} \times W$ by 


$$
g_{t}:(z, u) \in D(V, F) \mapsto\left(z\left(t Y z+E_{p}\right)^{-1}, u\right) \in R^{c} \times W
$$

Then we can easily verify that

$$
\left.\operatorname{Im}\left(z\left(t Y z+E_{p}\right)^{-1}\right)={ }^{t} \overline{\left(t Y z+E_{p}\right.}\right)^{-1} \operatorname{Im} z\left(t Y z+E_{p}\right)^{-1}
$$

and

$$
\left.{ }^{t} \overline{\left(t Y z+E_{p}\right.}\right)^{-1} F(u, u)\left(t Y z+E_{p}\right)^{-1}=F(u, u)
$$

for each $u \in W$. Therefore the map $g_{t}$ is a one-parameter group of transformations of $D(V, F)$ and the vector field $X$ induced by $g_{t}$ belongs to $g_{1}$. Furthermore we have $\xi(X)=\left(\begin{array}{ll}0 & 0 \\ Y & 0\end{array}\right)$. Considering Proposition 2.8 we can identify $g_{1}$ with the vector space $H(p-q, C)$.

Now we consider the case (ii). If $r_{1}(1)=r_{2}(1)=p$, then the Siegel domain $D(V, F)$ is the one given in (2) of Lemma 3.1. Thus we get $\mathfrak{g}_{h}=g_{a}$. We suppose that $r_{1}(1)=p$ and $r_{2}(1)<p$. We put $t_{0}=\min \{t$ $\in\left[1, s_{2}\right] ; t$ is an integer such that $\left.r_{2}(t)=p\right\}$ and define the subspaces $W_{1}$ and $W_{2}$ of $W$ by

$$
\begin{aligned}
& W_{1}=\left\{u=u^{(1)}+u^{(2)} \in W ; u_{i j}^{(2)}=0 \quad \text { if } j<t_{0}\right\} \\
& W_{2}=\left\{u=u^{(1)}+u^{(2)} \in W ; u^{(1)}=0, u_{i j}^{(2)}=0 \quad \text { if } j \geq t_{0}\right\} .
\end{aligned}
$$

Then we can see that

$$
W \doteq W_{1}+W_{2} \text { (direct sum) and } F\left(W_{1}, W_{2}\right)=(0) .
$$

The Siegel domain $D\left(V, F_{1}\right)$ in $R^{c} \times W_{1}$ is isomorphic to the one given in (2) of Lemma 3.1. Thus we get $\mathfrak{g}_{1 / 2}^{(1)}=(0)$.

For the Siegel domain $D\left(V, F_{2}\right)$ in $R^{c} \times W_{2}$, it can be seen that the linear closure of the set $\left\{F_{2}(u, u) ; u \in W_{2}\right\}$ in $R$ coincides with the proper subspace $\left(\begin{array}{cc}H(q, C) & 0 \\ 0 & 0\end{array}\right)$ of $R$, where $q=r_{2}\left(t_{0}-1\right)$ (cf. [4]). From Lemma 3.2 it follows that $\mathfrak{g}_{1 / 2}^{(2)}=(0)$. By Corollary 2.7 we have $g_{1 / 2}=(0)$. Applying Proposition 2.2 to the non-degenerate Siegel domain $D(V, F)$, we get $\mathrm{g}_{h}=\mathrm{g}_{a}$.

If $r_{1}(1) \neq p$ and $r_{2}(1)=p$, then the fact $\mathfrak{g}_{h}=\mathfrak{g}_{a}$ can be analogously obtained.

Now we suppose that $r_{1}(1) \neq p$ and $r_{2}(1) \neq p$. We put $t_{i}=$ $\min \left\{t \in\left[1, s_{i}\right] ; t\right.$ is an integer such that $\left.r_{i}(t)=p\right\} \quad(i=1,2)$ and define the subspaces $W_{i}(i=1,2)$ of $W$ by 


$$
W_{1}=\left\{u=u^{(1)}+u^{(2)} \in W ; u_{i j}^{(1)}=0 \text { if } j<t_{1}, u_{i j}^{(2)}=0 \text { if } j<t_{2}\right\}
$$

and

$$
W_{2}=\left\{u=u^{(1)}+u^{(2)} \in W ; u_{i j}^{(1)}=0 \text { if } j \geq t_{1}, u_{i j}^{(2)}=0 \text { if } j \geq t_{2}\right\} .
$$

Then we have

$$
W=W_{1}+W_{2} \text { (direct sum) and } F\left(W_{1}, W_{2}\right)=(0) \text {. }
$$

It is easy to see that the Siegel domain $D\left(V, F_{1}\right)$ in $R^{c} \times W_{1}$ is isomorphic to the one given in (2) of Lemma 3.1. Thus we have $\mathfrak{g}_{1 / 2}^{(1)}=(0)$. And for the Siegel domain $D\left(V, F_{2}\right)$ in $R^{c} \times W_{2}$, the linear closure of the set $\left\{F_{2}(u, u) ; u \in W_{2}\right\}$ in $R$ coincides with the proper subspace $\left(\begin{array}{cc}H(q, C) & 0 \\ 0 & 0\end{array}\right)$ of $R$, where $q=\max \left(r_{1}\left(t_{1}-1\right), r_{2}\left(t_{2}-1\right)\right.$ ) (cf. [4]). Hence by Lemma 3.2 we get $\mathfrak{g}_{1 / 2}^{(2)}=(0)$. From Corollary 2.7 it follows that $\mathfrak{g}_{1 / 2}=(0)$. Using Proposition 2.2 we conclude that $\mathfrak{g}_{h}=\mathfrak{g}_{a}$. q.e.d.

THEOREM 3.5.) If $r_{1}\left(s_{1}\right)<p$ and $r_{2}\left(s_{2}\right)=p$, then the subspaces $\mathfrak{g}_{1 / 2}$ and $\mathfrak{g}_{1}$ of $\mathfrak{g}_{h}$ are given as follows;

$\mathrm{g}_{1 / 2}$ is isomorphic to the real vector space $M\left(s_{0}, p-q ; C\right)$,

$\mathrm{g}_{1}$ is isomorphic to the vector space $H(p-q, C)$, where $s_{0}=s_{2}-t_{0}+1, q=\max \left(r_{1}\left(s_{1}\right), r_{2}\left(t_{0}-1\right)\right)$ and $t_{0}=\min \left\{t \in\left[1, s_{2}\right]\right.$; $t$ is an integer such that $\left.r_{2}(t)=p\right\}$, and $r_{2}\left(t_{0}-1\right)$ means zero if $t_{0}=1$.

Proof. We define the subspaces $W_{1}$ and $W_{2}$ of $W$ by

$$
\begin{aligned}
& W_{1}=\left\{u=u^{(1)}+u^{(2)} \in W ; u^{(1)}=0, u_{i j}^{(2)}=0 \text { if } j<t_{0}\right\}, \\
& W_{2}=\left\{u=u^{(1)}+u^{(2)} \in W ; u_{i j}^{(2)}=0 \text { if } j \geq t_{0}\right\} .
\end{aligned}
$$

Then we can see that

$$
W=W_{1}+W_{2} \text { (direct sum) and } F\left(W_{1}, W_{2}\right)=(0) .
$$

If $W_{2}=(0)$, then $D(V, F)$ is the classical domain of type (I) (cf. [10], Chap. 2).*) Therefore we consider the case $W_{2} \neq(0)$.

The Siegel domain $D\left(V, F_{2}\right)$ in $R^{c} \times W_{2}$ is degenerate and the linear closure of the set $\left\{F_{2}(u, u) ; u \in W_{2}\right\}$ in $R$ coincides with the proper sub-

4) Nakajima [18] calculated the dimensions of $g_{1 / 2}$ and $g_{1}$ of this theorem by using different methods.

*) By the following decomposition of the Lie algebra $g_{h}{ }^{(1)}$, we can see that the theorem is valid for this case. 
space $\left(\begin{array}{cc}H(q, C) & 0 \\ 0 & 0\end{array}\right)$ of $R$ (cf. [4]). Hence, by Lemma 3.2 we get $g_{1 / 2}^{(2)}=(0)$.

On the other hand, the Siegel domain $D\left(V, F_{1}\right)$ in $R^{c} \times W_{1}$ is the classical domain of type (I). The Lie algebra $g_{h}^{(1)}$ can be identified with $\mathfrak{H} \mathfrak{u}\left(s_{0}+p, p\right)$ as follows (cf. [10], Chap. 2, §6);

$$
\begin{aligned}
& \mathfrak{g}_{h}^{(1)}=\mathfrak{s} \mathfrak{u}\left(s_{0}+p, p\right) \\
& =\left\{\left(\begin{array}{lll}
A_{11} & A_{12} & A_{13} \\
A_{21} & A_{22} & A_{23} \\
A_{31} & A_{32} & A_{33}
\end{array}\right) ; \begin{array}{l}
A_{33}=-{ }^{t} \bar{A}_{11} \in \mathfrak{g l}(p, C), i^{t} A_{23}, A_{32} \in \mathfrak{H}\left(s_{0}\right) \\
A_{13}, A_{31} \in H(p, C)
\end{array}\right\} \\
& \left(\bmod \left\{i \theta E_{2 p+s_{0}} ; \theta \in \boldsymbol{R}\right\}\right) \text {. } \\
& \mathfrak{g}_{-1}^{(1)}=\left(\begin{array}{ccc}
0 & 0 & H(p, C) \\
0 & 0 & 0 \\
0 & 0 & 0
\end{array}\right), \quad \mathfrak{g}_{-1 / 2}^{(1)}=\left\{\left(\begin{array}{ccc}
0 & C & 0 \\
0 & 0 & i^{t} \bar{C} \\
0 & 0 & 0
\end{array}\right) ; C \in M\left(p, s_{0} ; C\right)\right\}, \\
& \mathfrak{g}_{1}^{(1)}=\left(\begin{array}{ccc}
0 & 0 & 0 \\
0 & 0 & 0 \\
H(p, C) & 0 & 0
\end{array}\right), \quad \mathfrak{g}_{1 / 2}^{(1)}=\left\{\left(\begin{array}{ccc}
0 & 0 & 0 \\
D & 0 & 0 \\
0 & -i^{t} \bar{D} & 0
\end{array}\right) ; D \in M\left(s_{0}, p ; C\right)\right\} \text {, } \\
& \mathfrak{g}_{0}^{(1)}=\left\{\left(\begin{array}{rrr}
A & 0 & 0 \\
0 & B & 0 \\
0 & 0 & -{ }^{t} \frac{0}{A}
\end{array}\right) ; A \in \mathfrak{g l}(p, C), B \in \mathfrak{H}\left(s_{0}\right)\right\}
\end{aligned}
$$

$\left(\bmod \left\{i \theta E_{2 p+s_{0}} ; \theta \in \boldsymbol{R}\right\}\right)$.

First we note that for

$$
g=\left(\begin{array}{ccc}
E_{p} & 0 & 0 \\
D & E_{s_{0}} & 0 \\
-\frac{1}{2} i^{t} \bar{D} D & -i^{t} \bar{D} & E_{p}
\end{array}\right) \in \exp g_{1 / 2}^{(1)}
$$

and

$$
h=\left(\begin{array}{lll}
E_{p} & 0 & 0 \\
0 & E_{s_{0}} & 0 \\
Y & 0 & E_{p}
\end{array}\right) \in \exp \mathfrak{g}_{1}^{(1)},
$$

$g$ and $h$ act on $D\left(V, F_{1}\right)$ as follows (cf. [10]);

$$
g\left(z, u_{1}\right)=\left(z^{\prime}, u_{1}^{\prime}\right) \text { and } h\left(z, u_{1}\right)=\left(z\left(Y z+E_{p}\right)^{-1},{ }^{t}\left(Y z+E_{p}\right)^{-1} u_{1}\right),
$$

where

$$
z^{\prime}=z\left(-\frac{1}{2} i^{t} \bar{D} D z-i^{t} \bar{D}^{t} u_{1}+E_{p}\right)^{-1}
$$

and

$$
u_{1}^{\prime}={ }^{t}\left(-\frac{1}{2} i^{t} \bar{D} D z-i^{t} \bar{D}^{t} u_{1}+E_{p}\right)^{-1}\left({ }^{t} z^{t} D+u_{1}\right)
$$


for each $\left(z, u_{1}\right) \in D\left(V, F_{1}\right)$.

Now we show that if $\tilde{A}$ belongs to $\rho\left(g_{0}\right)(A \in \mathfrak{g l}(p, C))$, then $A$ must be of the form (3.5). In fact, there exists $B \in \mathfrak{g l}(W)$ such that $(\tilde{A}, B)$ satisfies the condition: $\tilde{A} F(u, u)=F(B u, u)+F(u, B u)$ for every $u \in W$. Putting $u=u_{2} \in W_{2}$ we have

$$
\tilde{A} F\left(u_{2}, u_{2}\right)=F\left(B u_{2}, u_{2}\right)+F\left(u_{2}, B u_{2}\right),
$$

which implies

$$
A F_{2}\left(u_{2}, u_{2}\right)+F_{2}\left(u_{2}, u_{2}\right)^{t} \bar{A}=F_{2}\left(\left(B u_{2}\right)_{2}, u_{2}\right)+F_{2}\left(u_{2},\left(B u_{2}\right)_{2}\right) .
$$

Therefore by the same considerations as in Lemma 3.2 it follows that $A$ must be of the form (3.5). By Proposition 2.6 we have

$$
\Phi_{-\lambda}\left(g_{-\lambda}\right)=g_{-\lambda}^{(1)} \quad\left(\lambda=1, \frac{1}{2}\right)
$$

and

$$
\Phi_{0}\left(\mathfrak{g}_{0}\right)=\left\{\left(\begin{array}{rrr}
A & 0 & 0 \\
0 & B & 0 \\
0 & 0 & -{ }^{t} \frac{A}{A}
\end{array}\right) \in \mathfrak{g}_{0}^{(1)} ; \tilde{A} \in \rho\left(\mathfrak{g}_{0}\right)\right\}
$$

Now we want to show that

$$
\Phi_{1 / 2}\left(g_{1 / 2}\right)=\left\{\left(\begin{array}{ccc}
0 & 0 & 0 \\
D & 0 & 0 \\
0 & -i^{t} \bar{D} & 0
\end{array}\right) \in \mathfrak{g}_{1 / 2}^{(1)} ; D=\left(0, D_{1}\right), D_{1} \in M\left(s_{0}, p-q ; C\right)\right\}
$$

Let $X \in g_{1 / 2}$. Then by (2) of Proposition 2.6 $\Phi_{1 / 2}(X)$ belongs to $\mathfrak{g}_{1 / 2}^{(1)}$. Thus, there exists $D \in M\left(s_{0}, p ; C\right)$ such that

$$
\Phi_{1 / 2}(X)=\left(\begin{array}{ccc}
0 & 0 & 0 \\
D & 0 & 0 \\
0 & -i^{t} \bar{D} & 0
\end{array}\right)
$$

From (1) and (4) of Proposition 2.6 it follows that $\left[\mathfrak{g}_{-1 / 2}^{(1)}, \Phi_{1 / 2}(X)\right]$ belongs to $\Phi_{0}\left(\mathrm{~g}_{0}\right)$. So, for each $C \in M\left(p, s_{0} ; C\right)$,

$$
\left[\left(\begin{array}{rrr}
0 & C & 0 \\
0 & 0 & i^{t} \bar{C} \\
0 & 0 & 0
\end{array}\right), \quad\left(\begin{array}{ccc}
0 & 0 & 0 \\
D & 0 & 0 \\
0 & -i^{t} \bar{D} & 0
\end{array}\right)\right] \text { belongs to } \Phi_{0}\left(\mathfrak{g}_{0}\right)
$$

Therefore $\widetilde{C D}$ is contained in $\rho\left(\mathfrak{g}_{0}\right)$. Thus $C D$ must be of the form (3.5), which implies that $D$ must be of the form (3.9). 
Conversely let $D\left(\in M\left(s_{0}, p ; C\right)\right)$ be of the form (3.9). We define the map $g_{t}(t \in \boldsymbol{R})$ of $D(V, F)$ into $R^{c} \times W$ by

$$
g_{t}:\left(z, u_{1}+u_{2}\right) \in D\left(V, F^{\prime}\right) \mapsto\left(z^{\prime}, u_{1}^{\prime}+u_{2}^{\prime}\right) \in R^{c} \times W,
$$

where

$$
\begin{aligned}
& z^{\prime}=z\left(-\frac{1}{2} i t^{2}{ }^{t} \bar{D} D-i t^{t} \bar{D}^{t} u_{1}+E_{p}\right)^{-1}, \\
& u_{1}^{\prime}={ }^{t}\left(-\frac{1}{2} i t^{2} t \bar{D} D z-i t^{t} \bar{D}^{t} u_{1}+E_{p}\right)^{-1}\left(t^{t} z^{t} D+u_{1}\right), \\
& u_{2}^{\prime}=u_{2} .
\end{aligned}
$$

Then, by elementary calculations we can verify that

$$
\operatorname{Im} z^{\prime}-F\left(u^{\prime}, u^{\prime}\right)={ }^{t} \bar{Q}(\operatorname{Im} z-F(u, u)) Q,
$$

where $Q=\left(-\frac{1}{2} i t^{2}{ }^{t} \bar{D} D z-i t^{t} \bar{D}^{t} u_{1}+E_{p}\right)^{-1}, u=u_{1}+u_{2}$ and $u^{\prime}=u_{1}^{\prime}+u_{2}^{\prime}$. Therefore the map $g_{t}$ is a one-parameter group of transformations of $D(V, F)$. Let $X$ be the vector field induced by $g_{t}$. Then it is obvious that $X$ belongs to $g_{1 / 2}$ and $\Phi_{1 / 2}(X)=\left(\begin{array}{ccc}0 & 0 & 0 \\ D & 0 & 0 \\ 0 & -i^{t} \bar{D} & 0\end{array}\right)$. By (2) of Proposition 2.6 we have proved that $g_{1 / 2}$ is isomorphic to the real vector space $M\left(s_{0}, p-q ; C\right)$.

Now we determine $g_{1}$. We can show

$$
\Phi_{1}\left(g_{1}\right)=\left\{\left(\begin{array}{lll}
0 & 0 & 0 \\
0 & 0 & 0 \\
Y & 0 & 0
\end{array}\right) \in \mathfrak{g}_{1}^{(1)} ; Y=\left(\begin{array}{cc}
0 & 0 \\
0 & y
\end{array}\right), y \in H(p-q ; C)\right\} .
$$

In fact, let $X \in \mathfrak{g}_{1}$. Then by (3) of Proposition 2.6 $\Phi_{1}(X)$ belongs to $\mathfrak{g}_{1}^{(1)}$. So, there exists $Y \in H(p, C)$ such that

$$
\Phi_{1}(X)=\left(\begin{array}{lll}
0 & 0 & 0 \\
0 & 0 & 0 \\
Y & 0 & 0
\end{array}\right) .
$$

From the condition $\left[\mathfrak{g}_{-1}, X\right] \subset \mathfrak{g}_{0}$ and (4) of Proposition 2.6 it follows that for each $B \in H(p, C)$,

$$
\left[\left(\begin{array}{lll}
0 & 0 & B \\
0 & 0 & 0 \\
0 & 0 & 0
\end{array}\right),\left(\begin{array}{lll}
0 & 0 & 0 \\
0 & 0 & 0 \\
Y & 0 & 0
\end{array}\right)\right] \text { belongs to } \Phi_{0}\left(\mathfrak{g}_{0}\right) .
$$

Hence, $\widetilde{B Y}$ belongs to $\rho\left(\mathrm{g}_{0}\right)$, which implies that $B Y$ must be of the form 
(3.5). Therefore $Y$ must be of the form (3.10). Conversely let $Y(\in H(p, C))$ be of the form (3.10). We define the map $h_{t}(t \in R)$ of $D(V, F)$ into $R^{c} \times W$ by

$$
h_{t}:\left(z, u_{1}+u_{2}\right) \in D(V, F) \mapsto\left(z^{\prime}, u_{1}^{\prime}+u_{2}^{\prime}\right) \in R^{c} \times W,
$$

where $z^{\prime}=z\left(t Y z+E_{p}\right)^{-1}, u_{1}^{\prime}={ }^{t}\left(t Y z+E_{p}\right)^{-1} u_{1}$ and $u_{2}^{\prime}=u_{2}$. Then we can verify that

$$
\operatorname{Im} z^{\prime}-F\left(u^{\prime}, u^{\prime}\right)={ }^{t}\left(\overline{t Y z+E_{p}}\right)^{-1}(\operatorname{Im} z-F(u, u))\left(t Y z+E_{p}\right)^{-1},
$$

where $u=u_{1}+u_{2}, \quad u^{\prime}=u_{1}^{\prime}+u_{2}^{\prime} \in W$. Therefore the map $h_{t}$ is a oneparameter group of transformations of $D(V, F)$ and $h_{t}$ induces a vector field $X \in \mathfrak{g}_{1}$ such that $\Phi_{1}(X)=\left(\begin{array}{lll}0 & 0 & 0 \\ 0 & 0 & 0 \\ Y & 0 & 0\end{array}\right)$. Thus, by (3) of Proposition 2.6 we have proved that $g_{1}$ is isomorphic to the vector space $H(p-q, C)$.

Remark. If $r_{1}\left(s_{1}\right)=p$ and $r_{2}\left(s_{2}\right)<p$, then the Siegel domain $D(V, F)$ is isomorphic to the one given in the above theorem. If $s_{1}=s_{2}=1$, $r_{1}(1)=p-1$ and $r_{2}(1)=p$, then the fact $\operatorname{dim} g_{1 / 2}=2$ was proved by Sudo [12] by using different methods.

3.5. In this paragraph we treat the Siegel domains of type II over the cone $V=H(p, K)(p \geq 2)$.

Let $s$ be a positive integer and $r(t)$ be a non-decreasing integer valued function defined on an interval $[1, s]$ such that $1 \leq r(1), r(s) \leq 2 p$. We denote by $W$ the complex vector space of all complex $2 p \times s$-matrices $u=\left(u_{i j}\right)$ such that $u_{i j}=0$ if $i>r(j)$. We put $F(u, v)=\frac{1}{2}\left(u^{t} \bar{v}+J \bar{v}^{t} u^{t} J\right)$ for $u, v \in W$. Then it is known in [10] that the map $F$ is a $V$-hermitian form on $W$ and the Siegel domain $D(V, F)$ is homogeneous. Furthermore it was proved in [4] that the domain $D(V, F)$ is non-degenerate if and only if $r(s)=2 p$ or $2 p-1$.

THEOREM 3.6.5) (i) If a Siegel domain $D(V, F)$ mensioned above is degenerate, then the subspaces $\mathfrak{g}_{1 / 2}$ and $\mathfrak{g}_{1}$ of $\mathfrak{g}_{h}$ are given by

$\mathrm{g}_{1 / 2}=(0)$,

$\mathfrak{g}_{1}$ is isomorphic to the vector space $H(p-q, K)$, where $q=[(r(s)+1) / 2]$.

5) Nakajima [18] calculated the dimensions of $g_{1 / 2}$ and $g_{1}$ of this theorem by using different methods. 
(ii) If $s \geq 2$ and $r(1)=2 p$, or if $s \geq 3$ and there exists an integer $t_{0}$ such that $1<t_{0} \leq s-1, r\left(t_{0}\right)=2 p$ and $r\left(t_{0}-1\right) \leq 2 p-2$, then $\mathfrak{g}_{h}=\mathfrak{g}_{a}$.

Proof. First we consider the case (i). The linear closure of the set $\{F(u, u) ; u \in W\}$ in $R$ coincides with the proper subspace $\left(\begin{array}{cc}H(q, K) & 0 \\ 0 & 0\end{array}\right)$ of $R$, where $q=[(r(s)+1) / 2]$ (cf. [4]). Hence by Lemma 3.2 we have $\mathrm{g}_{1 / 2}=(0)$.

We determine $g_{1}$. Now, we consider the tube domain $D^{\prime}$ associated with $D\left(V, F^{\prime}\right)$ (cf. (2.9)). Then it is known in [10] that $D^{\prime}$ is the classical domain of type (II). The Lie algebra $\mathfrak{g}_{h}^{\prime}=\mathfrak{g}_{-1}^{\prime}+\mathfrak{g}_{0}^{\prime}+\mathfrak{g}_{1}^{\prime}$ of all infinitesimal automorphisms of $D^{\prime}$ can be identified with $3_{0} 0^{*}(4 p)$ as follows (cf. [10], Chap. 2, §7);

$$
\begin{aligned}
\mathfrak{g}_{h}^{\prime} & =\mathfrak{S}_{0}^{*}(4 p) \\
& =\left\{\left(\begin{array}{cr}
A & B \\
C & -{ }^{t} \bar{A}
\end{array}\right) ; A \in \operatorname{gr}(2 p, C), A J=J \bar{A}, B, C \in H(p, \boldsymbol{K})\right\}, \\
\mathfrak{g}_{-1}^{\prime} & =\left(\begin{array}{cc}
0 & H(p, \boldsymbol{K}) \\
0 & 0
\end{array}\right), \quad \mathfrak{g}_{1}^{\prime}=\left(\begin{array}{cc}
0 & 0 \\
H(p, \boldsymbol{K}) & 0
\end{array}\right), \\
\mathfrak{g}_{0}^{\prime} & =\left\{\left(\begin{array}{cc}
A & 0 \\
0 & -{ }^{t} \frac{t}{A}
\end{array}\right) ; A \in \operatorname{gr}(2 p, C), A J=J \bar{A}\right\} .
\end{aligned}
$$

We note that $g=\left(\begin{array}{cc}E_{2 p} & 0 \\ Y & E_{2 p}\end{array}\right)\left(\in \exp \mathrm{g}_{1}^{\prime}\right)$ acts on $D^{\prime}$ by

$$
g: z \in D^{\prime} \mapsto z\left(Y z+E_{2 p}\right)^{-1} \in D^{\prime} .
$$

It can be easily seen that the image $\xi\left(g_{0}\right)$ of $g_{0}$ (cf. (2.10)) is the following subalgebra of $g_{0}^{\prime}$;

$$
\xi\left(\mathfrak{g}_{0}\right)=\left\{\left(\begin{array}{cc}
A & 0 \\
0 & -{ }^{t} \bar{A}
\end{array}\right) \in \mathfrak{g}_{0}^{\prime} ; \tilde{A} \in \rho\left(\mathfrak{g}_{0}\right)\right\} .
$$

We want to show that $\xi\left(\mathfrak{g}_{1}\right)$ coincides with the following subspace of $\mathfrak{g}_{1}^{\prime}$;

$$
\left\{\left(\begin{array}{ll}
0 & 0 \\
Y & 0
\end{array}\right) \in g_{1}^{\prime} ; Y=\left(\begin{array}{ll}
0 & 0 \\
0 & y
\end{array}\right), y \in H(p-q, K)\right\} .
$$

In fact, let $X \in \mathfrak{g}_{1}$. Then $\xi(X)$ belongs to $\mathfrak{g}_{1}^{\prime}$ and there exists $Y \in H(p, K)$ such that $\xi(X)=\left(\begin{array}{ll}0 & 0 \\ Y & 0\end{array}\right)$. On the other hand, $\xi\left(\mathfrak{g}_{-1}\right)=\mathfrak{g}_{-1}^{\prime}$. So, by the condition $\left[\mathfrak{g}_{-1}, X\right] \subset \mathrm{g}_{0}$ we have $\left[\mathrm{g}_{-1}^{\prime}, \xi(X)\right] \subset \xi\left(\mathrm{g}_{0}\right)$. Hence, for each $B \in$ $H(p, K), \widetilde{B Y}$ must be contained in $\rho\left(\mathrm{g}_{0}\right)$. Therefore $B Y$ must be of the 
form (3.6). Thus, $Y$ must be of the form (3.11). Conversely let $Y$ be an element in $H(p, K)$ of the form (3.11). We define the map $g_{t}(t \in \boldsymbol{R})$ of $D(V, F)$ into $R^{c} \times W$ by

$$
g_{t}:(z, u) \in D(V, F) \mapsto\left(z\left(t Y z+E_{2 p}\right)^{-1}, u\right) \in R^{c} \times W .
$$

Then we can verify that

$$
\left.\operatorname{Im}\left(z\left(t Y z+E_{2 p}\right)^{-1}\right)=\overline{{ }^{t}\left(t Y z+E_{2 p}\right.}\right)^{-1} \operatorname{Im} z\left(t Y z+E_{2 p}\right)^{-1}
$$

and

$$
\overline{t^{t}\left(\overline{z+E_{2 p}}\right)^{-1}} \boldsymbol{F}(u, u)\left(t Y z+E_{2 p}\right)^{-1}=F(u, u) .
$$

Therefore the map $g_{t}$ is a one-parameter group of transformations of $D(V, F)$, and $g_{t}$ induces a vector field $X \in \mathfrak{g}_{1}$ such that $\xi(X)=\left(\begin{array}{ll}0 & 0 \\ Y & 0\end{array}\right)$. Thus, by the fact $g_{1 / 2}=(0)$ and Proposition $2.8 g_{1}$ can be identified with the vector space $H(p-q, K)$.

Now we consider the case (ii). If $r(1)=2 p$, then the complex vector space $W$ coincides with $M(2 p, s ; C)$ and the Siegel domain $D(V, F)$ is the one given in (3) of Lemma 3.1. So, we have $g_{h}=g_{a}$. We proceed to the second case. We define the subspaces $W_{1}$ and $W_{2}$ of $W$ by

$$
W_{1}=\left\{u=\left(u_{i j}\right) \in W ; u_{i j}=0 \text { if } j<t_{0}\right\}
$$

and

$$
W_{2}=\left\{u=\left(u_{i j}\right) \in W ; u_{i j}=0 \text { if } j \geq t_{0}\right\}
$$

Then we have

$$
W=W_{1}+W_{2} \text { (direct sum) and } F\left(W_{1}, W_{2}\right)=(0) .
$$

The vector space $W_{1}$ is isomorphic to $M\left(2 p, s-t_{0}+1 ; C\right)$ and the Siegel domain $D\left(V, F_{1}\right)$ in $R^{c} \times W_{1}$ is isomorphic to the one given in (3) of Lemma 3.1. Thus, we have $\mathfrak{g}_{1 / 2}^{(1)}=(0)$. For the Siegel domain $D\left(V, F_{2}\right)$ in $R^{c} \times W_{2}$, by our assumption $r\left(t_{0}-1\right) \leq 2 p-2$ the linear closure of the set $\left\{F_{2}(u, u) ; u \in W_{2}\right\}$ in $R$ coincides with the proper subspace $\left(\begin{array}{cc}H(q, K) & 0 \\ 0 & 0\end{array}\right)$ of $R$, where $q=\left[\left(r\left(t_{0}-1\right)+1\right) / 2\right]$ (cf. [4]). Thus, by Lemma 3.2 we get $g_{1 / 2}^{(2)}=(0)$. It follows from Corollary 2.7 that $g_{1 / 2}=(0)$. Applying Proposition 2.2 to the non-degenerate Siegel domain $D(V, F)$, we conclude that $g_{h}=g_{a}$.

q.e.d. 


\section{§ 4. Homogeneous Siegel domains over circular cones}

In this section, we will study how to construct all homogeneous non-degenerate Siegel domains over circular cones and study their equivalence. We omit the terminology "of type II of rank 2", since we consider here exclusively $N$-algebras of type II of rank 2 .

4.1. We will recall some of definitions and results about $N$-algebras and skeletons due to Kaneyuki and Tsuji [5] in the case of rank 2.

Let $N$ be a finite dimensional algebra over the real number field. Suppose that $N$ is the direct sum of the bigraded subspaces $N_{i j}(1 \leq i$ $<j \leq 3$ ) and that $N$ is equipped with a positive definite inner product $\langle$,$\rangle . Let j$ be a linear endomorphism of the subspace $N_{13}+N_{23}$ of $N$. Then the triple $(N,\langle\rangle, j$,$) is called an N$-algebra $\left.{ }^{6}\right)$ if the following conditions are satisfied;

$$
\begin{gathered}
N_{13} \neq(0) \quad \text { or } \quad N_{23} \neq(0), \\
N_{12} N_{23} \subset N_{13}, N_{i j} N_{k l}=(0) \quad \text { if } j \neq k, \\
\left\langle N_{i j}, N_{k l}\right\rangle=0 \quad \text { if } i \neq k \text { or } j \neq l, \\
j N_{i 3}=N_{i 3} \quad(i=1,2), j^{2}=-1, \\
\langle j a, j b\rangle=\langle a, b\rangle \quad \text { for } a, b \in N_{13}+N_{23}, \\
j\left(a_{12} a_{23}\right)=a_{12} j\left(a_{23}\right), \\
\text { for every } a_{12}, b_{12} \in N_{12} \text { and } a_{23}, b_{23} \in N_{23}, \\
\left\langle a_{12} a_{23}, b_{12} b_{23}\right\rangle+\left\langle a_{12} b_{23}, b_{12} a_{23}\right\rangle=2\left\langle a_{12}, b_{12}\right\rangle\left\langle a_{23}, b_{23}\right\rangle .
\end{gathered}
$$

Remark. Let $(N,\langle\rangle, j$,$) be an N$-algebra with $\operatorname{dim} N_{12} \cdot \operatorname{dim} N_{23} \neq 0$. Then the following condition is satisfied; $\max \left(\operatorname{dim} N_{12}, \operatorname{dim} N_{23}\right) \leq \operatorname{dim} N_{13}$ (cf. [5]).

A figure $\mathbb{S}$ in the plane is called a connected 2-skeleton (of type II) if $\mathfrak{S}$ is one of the following $\mathfrak{S}_{1}$ or $\mathfrak{S}_{2}$;
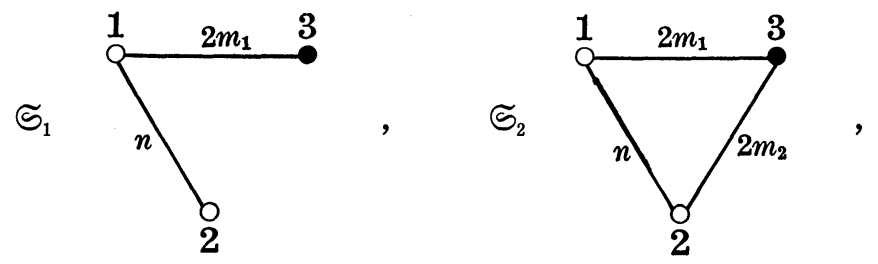

6) This definition is slightly different from that of [5], but these are equivalent. 
where $n$ and $m_{1}$ in $\widetilde{S}_{1}$ are positive integers, and $n, m_{1}, m_{2}$ in $\mathfrak{S}_{2}$ are positive integers such that $\max \left(n, 2 m_{2}\right) \leq 2 m_{1}$.

Let $(N,\langle\rangle, j$,$) be an N$-algebra. Then it is said that $(N,\langle\rangle, j$, corresponds to $\mathfrak{S}_{1}\left(\right.$ resp. $\mathfrak{S}_{2}$ ) if $\operatorname{dim} N_{12}=n, \operatorname{dim} N_{23}=0$ and $\operatorname{dim} N_{13}=$ $2 m_{1}$ (resp. $\operatorname{dim} N_{12}=n, \operatorname{dim} N_{23}=2 m_{2}$ and $\operatorname{dim} N_{13}=2 m_{1}$ ). In this case, $\mathbb{S}_{1}\left(\right.$ resp. $\left.\mathfrak{S}_{2}\right)$ is called the diagram of $(N,\langle\rangle, j$,$) .$

Let $(N,\langle\rangle, j$,$) and \left(N^{\prime},\langle,\rangle^{\prime}, j^{\prime}\right)$ be two $N$-algebras which correspond to the skeletons $\mathfrak{S}_{1}$ or $\mathfrak{S}_{2}$. Then $(N,\langle\rangle, j$,$) is said to be$ isomorphic to $\left(N^{\prime},\langle,\rangle^{\prime}, j^{\prime}\right)$ if there exists a bigrade-preserving algebra isomorphism $\varphi$ of $N$ onto $N^{\prime}$ such that

$$
\begin{aligned}
& \langle\varphi(a), \varphi(b)\rangle^{\prime}=\langle a, b\rangle, a, b \in N, \\
& \varphi \circ j=j^{\prime} \circ \varphi \quad \text { on } N_{13}+N_{23} .
\end{aligned}
$$

It follows immediately from the above definition that if two $\mathrm{N}$-algebras which correspond to the skeletons $\mathfrak{S}_{1}$ or $\mathbb{S}_{2}$ are isomorphic, then their diagrams are the same one.

According to [5], [13], there is a one-to-one correspondence between the set of all (holomorphic) isomorphism classes of homogeneous Siegel domains of type II over circular cones and the set of all isomorphism classes of $N$-algebras whose diagrams are $\mathfrak{S}_{1}$ or $\mathfrak{S}_{2}$.

In what follows, for a Siegel domain $D(C(n+2), F)$ corresponding to an $N$-algebra whose diagram is $\widetilde{S}_{1}\left(\right.$ resp. $\left.\widetilde{S}_{2}\right)$, we say that $D(C(n+2), F)$ corresponds to $\mathfrak{S}_{1}$ (resp. $\mathfrak{S}_{2}$ ).

It is known in [5] that for given positive integers $n, m_{1}$, there exists a unique homogeneous Siegel domain which corresponds to $\mathfrak{S}_{1}$. Furthermore the explicit forms of these domains are found in [5], [10].

4.2. By the facts stated above we will consider the case of $\mathfrak{S}_{2}$.

DEFINITION 4.1. Let $\left\{T_{k}\right\}_{1 \leq k \leq n}$ be a system of $m_{1} \times m_{2}$-complex matrices $T_{k}(1 \leq k \leq n)$ satisfying the condition;

$$
{ }^{t} \bar{T}_{k} T_{l}+{ }^{t} \bar{T}_{l} T_{k}=2 \delta_{k l} E_{m_{2}} \quad(1 \leq k, l \leq n) .
$$

Let $\left\{T_{k}^{\prime}\right\}_{1 \leq k \leq n}$ be another system of $m_{1} \times m_{2}$-complex matrices satisfying (4.6). Then $\left\{T_{k}\right\}_{1 \leq k \leq n}$ is said to be equivalent to $\left\{T_{k}^{\prime}\right\}_{1 \leq k \leq n}$ if there exists a triple $\left(O_{1}, U_{1}, U_{2}\right) \in O(n) \times U\left(m_{1}\right) \times U\left(m_{2}\right)$ such that

$$
\left(T_{1}, \cdots, T_{n}\right)=U_{1}\left(T_{1}^{\prime}, \cdots, T_{n}^{\prime}\right)\left(O_{1} \otimes U_{2}\right)
$$


for the $m_{1} \times n m_{2}$-matrices $\left(T_{1}, \cdots, T_{n}\right)$ and $\left(T_{1}^{\prime}, \cdots, T_{n}^{\prime}\right)$.

From (4.7) it can be seen that the above "equivalence" is an equivalence-relation in the set of all systems satisfying (4.6).

Let $\left\{T_{k}\right\}_{1 \leq k \leq n}$ be a system of $m_{1} \times m_{2}$-matrices satisfying (4.6). Let $N_{12}$ be the euclidean space $\boldsymbol{R}^{n}$ with the inner product (, ) and $N_{k 3}$ be the complex euclidean space $C^{m_{k}}(k=1,2)$ with the hermitian inner product (, ). Let $N$ be the direct sum of real vector spaces $N_{i j}(1 \leq i$ $<j \leq 3$ ). Then for a fixed orthonormal base $\left\{e_{k}\right\}_{1 \leq k \leq n}$ of $N_{12}$, we define in $N$ an inner product $\langle$,$\rangle , a multiplication and a complex structure$ $j$ as follows;

$$
\begin{gathered}
\left\langle a_{12}+a_{23}+a_{13}, b_{12}+b_{23}+b_{13}\right\rangle \\
=\left(a_{12}, b_{12}\right)+\operatorname{Re}\left(a_{23}, b_{23}\right)+\operatorname{Re}\left(a_{13}, b_{13}\right), \\
a_{i j}, b_{i j} \in N_{i j} \quad(1 \leq i<j \leq 3) .
\end{gathered}
$$

$$
\begin{gathered}
e_{k} a_{23}=T_{k} a_{23} \text { holds in } N_{13}(1 \leq k \leq n) \text { and } a_{i j} a_{s t}=0 \text { if } j \neq s . \\
j a_{k 3}=i a_{k 3} \quad(k=1,2) .
\end{gathered}
$$

LEMMA 4.2. With respect to (4.8), (4.9) and (4.10) the vector space $N$ is an N-algebra which corresponds to $\mathfrak{S}_{2}$. Every $N$-algebra which corresponds to $\mathfrak{S}_{2}$ can be obtained in this way by taking some system satisfying (4.6).

Proof. It can be easily seen that $(N,\langle\rangle, j$,$) satisfies all the con-$ ditions but (4.4). Using (4.6), (4.8) and (4.9), we obtain

$$
\begin{aligned}
& \left\langle e_{k} a_{23}, e_{l} b_{23}\right\rangle+\left\langle e_{k} b_{23}, e_{l} a_{23}\right\rangle \\
& \quad=\operatorname{Re}\left(T_{k} a_{23}, T_{l} b_{23}\right)+\operatorname{Re}\left(T_{k} b_{23}, T_{l} a_{23}\right) \\
& \quad=\operatorname{Re}\left(\left({ }^{t} \bar{T}_{k} T_{l}+{ }^{t} \bar{T}_{l} T_{k}\right) a_{23}, b_{23}\right)=2 \delta_{k l} \operatorname{Re}\left(a_{23}, b_{23}\right) \\
& \quad=2\left\langle e_{k}, e_{l}\right\rangle\left\langle a_{23}, b_{23}\right\rangle,
\end{aligned}
$$

which implies (4.4). By Remark in the paragraph 4.1 it is obvious that $(N,\langle\rangle, j$,$) corresponds to \mathfrak{S}_{2}$. Hence the first assertion was proved.

Conversely let $(N,\langle\rangle, j$,$) be an N$-algebra which corresponds to $\mathfrak{S}_{2}$. Then by (4.1) and (4.2) we can identify $N_{13}$ (resp. $N_{23}$ ) with $C^{m_{1}}$ (resp. $\boldsymbol{C}^{m_{2}}$ ) as hermitian vector spaces. Let us identify $N_{12}$ with $\boldsymbol{R}^{n}$ as euclidean vector spaces and put $\left\{e_{k}\right\}_{1 \leq k \leq n}$ be an orthonormal base of $N_{12}$ $=\boldsymbol{R}^{n}$. Let $L_{k}$ denote the left multiplication by $e_{k}$ in $N$ (i.e., $L_{k}(x)=$ $e_{k} x$ for $\left.x \in N\right) \quad(1 \leq k \leq n)$. Then $L_{k}$ restricted to the subspace $N_{23}$ 
induces a complex linear mapping of $N_{23}$ into $N_{13}$ (cf. (4.3)). Hence, under the identification of $N_{i 3}$ with $C^{m_{i}}(i=1,2) L_{k}$ induces a complex $m_{1} \times m_{2}$-matrix $T_{k}$ such that $T_{k} a_{23}=e_{k} a_{23}(1 \leq k \leq n)$. On the other hand, (4.4) implies

$$
L_{k}^{*} L_{l}+L_{l}^{*} L_{k}=2 \delta_{k l} 1,
$$

where $*$ is the adjoint with respect to the inner product $\langle$,$\rangle . Thus,$ it follows that the system $\left\{T_{k}\right\}_{1 \leq k \leq n}$ satisfies the condition (4.6). q.e.d.

In view of the above lemma the system $\left\{T_{k}\right\}_{1 \leq k \leq n}$ is called the $a d$ missible system of $(N,\langle\rangle, j$,$) with respect to the orthonormal base$ $\left\{e_{k}\right\}_{1 \leq k \leq n}$.

LEMMA 4.3. Let $(N,\langle\rangle, j$,$) and \left(N^{\prime},\langle,\rangle^{\prime}, j^{\prime}\right)$ be two $N$-algebras which correspond to $\mathfrak{S}_{2}$. Let $\left\{e_{k}\right\}_{1 \leq k \leq n}$ (resp. $\left\{e_{k}^{\prime}\right\}_{1 \leq k \leq n}$ ) be an arbitrary orthonormal base of $N_{12}$ (resp. $N_{12}^{\prime}$ ) and let $\left\{T_{k}\right\}_{1 \leq k \leq n}$ (resp. $\left\{T_{k}^{\prime}\right\}_{1 \leq k \leq n}$ ) be the admissible system of $(N,\langle\rangle, j$,$) (resp. \left(N^{\prime},\langle,\rangle^{\prime}, j^{\prime}\right)$ ) with respect to $\left\{e_{k}\right\}_{1 \leq k \leq n}$ (resp. $\left.\left\{e_{k}^{\prime}\right\}_{1 \leq k \leq n}\right)$. Then $(N,\langle\rangle, j$,$) is isomorphic to \left(N^{\prime},\langle,\rangle^{\prime}, j^{\prime}\right)$ if and only if $\left\{T_{k}\right\}_{1 \leq k \leq n}$ is equivalent to $\left\{T_{k}^{\prime}\right\}_{1 \leq k \leq n}$.

Proof. Suppose that $(N,\langle\rangle, j$,$) is isomorphic to \left(N^{\prime},\langle,\rangle^{\prime}, j^{\prime}\right)$. Then from (4.5) it follows that there exists a triple $(f, g, h)$ of linear isometries;

$$
f: N_{12} \rightarrow N_{12}^{\prime}, \quad g: N_{23} \rightarrow N_{23}^{\prime}, \quad h: N_{13} \rightarrow N_{13}^{\prime}
$$

satisfying

$$
f\left(e_{k}\right) g\left(a_{23}\right)=h\left(e_{k} a_{23}\right)
$$

and

$$
h \circ j=j^{\prime} \circ h \text { on } N_{13} \text { and } g \circ j=j^{\prime} \circ g \text { on } N_{23} \text {. }
$$

Let $O=\left(\alpha_{l k}\right)$ be the orthogonal matrix of degree $n$ defined by $f\left(e_{k}\right)=$ $\sum \alpha_{l k} e_{l}^{\prime}(1 \leq k \leq n)$. Then (4.11) implies $\sum \alpha_{l k} e_{l}^{\prime} g\left(a_{23}\right)=h\left(e_{k} a_{23}\right)$. Hence, we have

$$
\sum \alpha_{l k} L_{l}^{\prime} \circ g=h \circ L_{k} \quad(1 \leq k \leq n) .
$$

From (4.12) it follows that $g$ (resp. $h$ ) induces a unitary matrix $G$ (resp. $H$ ) of degree $m_{2}$ (resp. $\left.m_{1}\right)$. Thus, (4.13) shows that $\sum \alpha_{l k} T_{l}^{\prime} G=H T_{k} \quad(1 \leq$ $k \leq n)$. From this we have

$$
\left(T_{1}^{\prime}, \cdots, T_{n}^{\prime}\right)(O \otimes G)=H\left(T_{1}, \cdots, T_{n}\right)
$$


Hence, $\left\{T_{k}\right\}_{1 \leq k \leq n}$, is equivalent to $\left\{T_{k}^{\prime}\right\}_{1 \leq k \leq n}$ (cf. Definition 4.1).

The converse of our assertion is analogously proved.

q.e.d.

4.3. It was proved in [5] that homogeneous Siegel domains and $\mathrm{N}$-algebras are in one-to-one correspondence. By considering the correspondence in detail in the rank 2 case, we will prove that every homogeneous non-degenerate Siegel domain $D(C(n+2), F)$ is constructed directly in terms of the system $\left\{T_{k}\right\}_{1 \leq k \leq n}$.

Let $(N,\langle\rangle, j$,$) be an N$-algebra whose diagram is $\mathfrak{S}_{2}$ and let $\left\{T_{k}\right\}_{1 \leq k \leq n}$ be the admissible system of $(N,\langle\rangle, j$,$) . Now we will construct the$ Siegel domain $D(C(n+2), F)$ which corresponds to $(N,<,>, j)$ in the sense of Corollary 2.7 in [5]. By Theorem 2.6 in [5] we can construct the $T$-algebra $\left(\mathfrak{U}=\sum_{1 \leq i, j \leq 3} \mathfrak{A}_{i j}, *, j\right)$ which corresponds to $(N,\langle\rangle, j$,$) as$ follows ;

$$
\mathfrak{U}_{i i}=R(1 \leq i \leq 3), \mathfrak{U}_{i j}=N_{i j}, \mathfrak{A}_{j i}=N_{i j}^{*}(1 \leq i<j \leq 3),
$$

where $*$ is an involutive linear endomorphism of $N_{i j}$ such that $* \circ j=$ $j \circ *$ on $N_{13}+N_{23}$. And the multiplications in $\mathfrak{A}$ have the following properties ;

$$
\begin{aligned}
& a_{i j} a_{j i}=\left\langle a_{i j}, a_{j i}^{*}\right\rangle \quad(1 \leq i<j \leq 3), \\
& \left\langle a_{13} a_{32}, e_{k}\right\rangle=\left\langle a_{13}, e_{k} a_{32}^{*}\right\rangle=\operatorname{Re}\left(a_{13}, T_{k} a_{32}^{*}\right),
\end{aligned}
$$

where $a_{i j} \in \mathfrak{A}_{i j}$.

We denote by $R(\mathfrak{O})$ the direct sum $\mathfrak{A}_{11}+\mathfrak{A}_{22}+\mathfrak{A}_{12}$ and denote by $W(\mathfrak{U})$ the direct sum $\mathfrak{A}_{13}+\mathfrak{A}_{23}\left(=C^{m_{1}}+C^{m_{2}}\right)$. We define the subset $V(N)$ of $R(\mathfrak{U})$ as

$$
V(N)=\left\{a=a_{11}+a_{22}+a_{12} \in R(\mathfrak{U}) ; a_{11}>0, a_{11} a_{22}-\left\langle a_{12}, a_{12}\right\rangle>0\right\}^{*)} .
$$

Then we can see that $V(N)$ is a homogeneous convex cone and actually isomorphic to $C(n+2)$ under the following linear isomorphim $f$ of $R(\mathfrak{U})$ onto $\boldsymbol{R}^{n+2}$;

$$
f: a=a_{11}+a_{22}+a_{12} \in R(\mathfrak{U}) \mapsto{ }^{t}\left(a_{11}, a_{22}, a_{12}^{1}, \cdots, a_{12}^{n}\right) \in \boldsymbol{R}^{n+2},
$$

where $a_{12}=\sum a_{12}^{k} e_{k}$.

We define the map $\boldsymbol{F}: \boldsymbol{C}^{m_{1}+m_{2}} \times \boldsymbol{C}^{m_{1}+m_{2}} \mapsto \boldsymbol{C}^{n+2}$ by putting $\boldsymbol{F}=$ ${ }^{t}\left(F^{1}, \cdots, F^{n+2}\right)$, where

*) By $a_{11} a_{22}$ we mean a usual multiplication of real numbers $a_{i i} \in \mathfrak{U}_{i i}=\boldsymbol{R}(i=1,2)$. 


$$
\begin{aligned}
F^{1}(u, v) & =\left(u_{1}, v_{1}\right), \quad F^{2}(u, v)=\left(u_{2}, v_{2}\right), \\
F^{k+2}(u, v) & =\frac{1}{2}\left\{\left(u_{1}, T_{k} v_{2}\right)+\left(T_{k} u_{2}, v_{1}\right)\right\} \quad(1 \leq k \leq n)
\end{aligned}
$$

for $u=u_{1}+u_{2}, v=v_{1}+v_{2} \in C^{m_{1}+m_{2}}=C^{m_{1}}+C^{m_{2}}$. Then we have

THEOREM 4.4." (i) For $F$ above, the domain $D(C(n+2), F)$ is a homogeneous non-degenerate Siegel domain.

(ii) Conversely every homogeneous non-degenerate Siegel domain $D(C(n$ $+2), F$ ) is constructed in the above way (4.16) by taking some system $\left\{T_{k}\right\}_{1 \leq k \leq n}$ satisfying (4.6).

(iii) Furthermore suppose that $D\left(C(n+2), F^{\prime}\right)$ is constructed by $\left\{T_{k}^{\prime}\right\}_{1 \leq k \leq n}$. Then $D(C(n+2), F)$ is holomorphically isomorphic to $D\left(C(n+2), F^{\prime}\right)$ if and only if $\left\{T_{k}\right\}_{1 \leq k \leq n}$ is equivalent to $\left\{T_{k}^{\prime}\right\}_{1 \leq k \leq n}$.

Proof. First we will show that the map $F$ defined by (4.16) is a $C(n+2)$-hermitian form on $C^{m_{1}}+C^{m_{2}}$ and the Siegel domain $D(C(n+2), F)$ thus constructed is the one which corresponds to $(N,\langle\rangle, j$,$) in the sense$ of [5]. By Theorem $A$ in [13], the homogeneous Siegel domain which corresponds to the $T$-algebra $(\mathscr{A}, *, j)$ is given by the following $V(N)$ hermitian form $\tilde{F}=\sum_{1 \leq k \leq l \leq 2} F_{k l}$ on $W(\mathfrak{U})$;

$$
F_{k l}(u, v)=\frac{1}{4}\left\{\left(u_{k 3} v_{l 3}^{*}+v_{k 3} u_{l 3}^{*}\right)+i\left(u_{k 3} j\left(v_{l 3}^{*}\right)+j\left(v_{k 3}\right) u_{l 3}^{*}\right)\right\}
$$

for $u=u_{13}+u_{23}, v=v_{13}+v_{23} \in W(\mathfrak{U})$.

Hence, by (4.14) we have

$$
\begin{aligned}
F_{k k}(u, v) & =\frac{1}{4}\left\{2\left\langle u_{k 3}, v_{k 3}\right\rangle+i\left(\left\langle u_{k 3}, j\left(v_{k 3}^{*}\right)^{*}\right\rangle+\left\langle j\left(v_{k 3}\right), u_{k 3}\right\rangle\right)\right\} \\
& =\frac{1}{2}\left\{\left\langle u_{k 3}, v_{k 3}\right\rangle+i\left\langle u_{k 3}, j\left(v_{k 3}\right)\right\rangle\right\}(\mathrm{by} * \circ j=j \circ *) \\
& =\frac{1}{2}\left\{\operatorname{Re}\left(u_{k 3}, v_{k 3}\right)+i \operatorname{Re}\left(u_{k 3}, i v_{k 3}\right)\right\}(\mathrm{by}(4.8)) \\
& =\frac{1}{2}\left(u_{k 3}, v_{k 3}\right) \quad(k=1,2) .
\end{aligned}
$$

And we have

$$
\begin{aligned}
\left\langle F_{12}(u, u), e_{k}\right\rangle & =\frac{1}{2}\left\langle u_{13} u_{23}^{*}, e_{k}\right\rangle+\frac{1}{4} i\left(\left\langle u_{13} j\left(u_{23}\right)^{*}, e_{k}\right\rangle+\left\langle j\left(u_{13}\right) u_{23}^{*}, e_{k}\right\rangle\right) \\
& =\frac{1}{2} \operatorname{Re}\left(u_{13}, T_{k} u_{23}\right)(\text { by }(4.14))
\end{aligned}
$$

which implies

$$
F_{12}(u, v)=\frac{1}{4} \sum_{1 \leq k \leq n}\left\{\left(u_{13}, T_{k} v_{23}\right)+\left(T_{k} u_{23}, v_{13}\right)\right\} e_{k} .
$$

7) If $m_{1}=m_{2}$ in $\varsigma_{2}$, then this construction is reduced to Pjateckii-Sapiro's [10]. 
We define the complex linear isomorphism $g$ of $W(\mathfrak{H})$ onto $\boldsymbol{C}^{m_{1}}+\boldsymbol{C}^{m_{2}}$ by

$$
g: u_{13}+u_{23} \in W(\mathfrak{Y}) \mapsto \frac{1}{\sqrt{2}} u_{13}+\frac{1}{\sqrt{2}} u_{23} \in C^{m_{1}}+C^{m_{2}} .
$$

Then we have

$$
f(\tilde{F}(u, v))=F(g(u), g(v)) \quad(u, v \in W(\mathfrak{U}), \text { cf. (4.15)) } .
$$

Thus, it can be seen that the map $F$ defined by (4.16) is a $C(n+2)$ hermitian form on $C^{m_{1}}+C^{m_{2}}$ and the Siegel domain $D(C(n+2), F)$ in $C^{n+2} \times C^{m_{1}+m_{2}}$ is linearly isomorphic to the Siegel domain $D(V(N), \tilde{F})$ in $R(\mathfrak{U})^{c} \times W(\mathfrak{U})$. Hence, the homogeneous Siegel domain $D(C(n+2), F)$ is the one which corresponds to $(N,\langle\rangle, j$,$) in the sense of Corollary 2.7$ in [5]. From Lemma 4.2 it follows that every homogeneous Siegel domain of type II over the cone $C(n+2)$ which corresponds to the skeleton $\widetilde{S}_{2}$ is constructed by (4.16) by taking some system $\left\{T_{k}\right\}_{1 \leq k \leq n}$ satisfying (4.6).

Now we will show that a homogeneous Siegel domain $D(C(n+2), F)$ is non-degenerate if and only if $D(C(n+2), F)$ corresponds to $\Im_{2}$. Suppose that $D(C(n+2), F)$ corresponds to $\widetilde{S}_{2}$. Then, as was proved above, $D(C(n+2), F)$ is constructed by (4.16) by some system $\left\{T_{k}\right\}_{1 \leq k \leq n}$ satisfying (4.6). The subset $\left\{\boldsymbol{F}(u, u) ; u \in \boldsymbol{C}^{m_{1}}+\boldsymbol{C}^{m_{2}}\right\}$ of $\boldsymbol{R}^{n+2}$ contains $n+2$ linearly independent vectors in $\boldsymbol{R}^{n+2}$. In fact, take unit vectors $u_{i} \in \boldsymbol{C}^{m_{i}}$ $(i=1,2)$ and put

$$
\begin{array}{r}
u^{1}=u_{1}+0, \quad u^{2}=0+u_{2}, \quad u^{k+2}=T_{k} u_{2}+u_{2} \in C^{m_{1}}+C^{m_{2}} \\
(1 \leq k \leq n) .
\end{array}
$$

Then we can verify that $\left\{F\left(u^{1}, u^{1}\right), F\left(u^{2}, u^{2}\right), \cdots, F\left(u^{n+2}, u^{n+2}\right)\right\}$ spans $R^{n+2}$. Suppose that $D(C(n+2), F)$ corresponds to $\widetilde{S}_{1}$. Then it was proved in [5], [10] that the $C(n+2)$-hermitian form $F$ on $C^{m_{1}}$ is given by

$$
F(u, v)={ }^{t}((u, v), 0, \cdots, 0) \quad\left(u, v \in C^{m_{1}}\right) .
$$

Hence $D(C(n+2), F)$ is degenerate.

Thus, the first and the second assertions of the theorem were proved. The last assertion follows immediately from Lemma 4.3. q.e.d.

\section{§5. The exceptional bounded symmetric domain of type (V)}

5.1. Let $\left\{T_{1}, T_{2}\right\}$ be a system satisfying the condition (4.6) and define 
an $m_{1} \times 2 m_{2}$-matrix $B$ as $B=\left(T_{1}, T_{2}\right)$. Then it follows from (4.6) that ${ }^{t} \bar{T}_{1} T_{2}$ is a skew-hermitian matrix of degree $m_{2}$, and we have

$$
{ }^{t} \bar{B} B=\left(\begin{array}{cc}
0 & 1 \\
-1 & 0
\end{array}\right) \otimes{ }^{t} \bar{T}_{1} T_{2}+E_{2 m_{2}} .
$$

LEMMA 5.1. Let $\left\{T_{1}, T_{2}\right\}$ and $\left\{T_{1}^{\prime}, T_{2}^{\prime}\right\}$ be two systems satisfying (4.6). Suppose that ${ }^{t} \bar{T}_{1} T_{2}$ (resp. ${ }^{t} \bar{T}_{1}^{\prime} T_{2}^{\prime}$ ) has eigenvalues $\left\{i \lambda_{1}, \cdots, i \lambda_{m_{2}}\right\}, \lambda_{1} \leq, \cdots$, $\leq \lambda_{m_{2}}$ (resp. $\left\{i \lambda_{1}^{\prime}, \cdots, i \lambda_{m_{2}}^{\prime}\right\}, \lambda_{1}^{\prime} \leq, \cdots, \lambda_{m_{2}}^{\prime}$ ). Then $\left\{T_{1}, T_{2}\right\}$ is equivalent to $\left\{T_{1}^{\prime}, T_{2}^{\prime}\right\} \quad$ if and only if $\left(\lambda_{1}, \cdots, \lambda_{m_{2}}\right)=\left(\lambda_{1}^{\prime}, \cdots, \lambda_{m_{2}}^{\prime}\right)$ or $\left(\lambda_{1}, \cdots, \lambda_{m_{2}}\right)=$ $\left(-\lambda_{m_{2}}^{\prime}, \cdots,-\lambda_{1}^{\prime}\right)$.

Proof. Suppose that $\left(\lambda_{1}, \cdots, \lambda_{m_{2}}\right)=\left(\lambda_{1}^{\prime}, \cdots, \lambda_{m_{2}}^{\prime}\right)$ or $\left(\lambda_{1}, \cdots, \lambda_{m_{2}}\right)=$ $\left(-\lambda_{m_{2}}^{\prime}, \cdots,-\lambda_{1}^{\prime}\right)$. Then there exists $U_{2} \in U\left(m_{2}\right)$ such that ${ }^{t} \bar{U}_{2}{ }^{t} \bar{T}_{1}^{\prime} T_{2}^{\prime} U_{2}=$ $\varepsilon^{t} \bar{T}_{1} T_{2}, \varepsilon= \pm 1$. Putting $B^{\prime \prime}=B^{\prime}\left(\left(\begin{array}{ll}1 & 0 \\ 0 & \varepsilon\end{array}\right) \otimes U_{2}\right)$, we have ${ }^{t} \bar{B}^{\prime \prime} B^{\prime \prime}={ }^{t} \bar{B} B$. Hence, by an analogous consideration as in Lemma 4.3 in [5], there exists $U_{1} \in U\left(m_{1}\right)$ satisfying $B=U_{1} B^{\prime \prime}$, that is, $B=U_{1} B^{\prime}\left(\left(\begin{array}{ll}1 & 0 \\ 0 & \varepsilon\end{array}\right) \otimes U_{2}\right)$. Therefore $\left\{T_{1}, T_{2}\right\}$ is equivalent to $\left\{T_{1}^{\prime}, T_{2}^{\prime}\right\}$ (cf. Definition 4.1). By making use of (5.1) we can easily prove the "only if" part.

q.e.d.

The following proposition is stated without proof in Pjateckii-Sapiro [10], but for the sake of completeness we prove it without using the theory of Clifford algebras.

Proposition 5.2. There exists a unique homogeneous Siegel domain (up to holomorphic equivalence) which corresponds to $\mathfrak{S}_{2}$ with $\left(n, m_{1}, m_{2}\right)$ $=(6,4,4)$. Furthermore this Siegel domain is constructed by the following system $\left\{T_{k}\right\}_{1 \leq k \leq 6}$;

$$
\begin{gathered}
T_{1}=E_{4}, \quad T_{2}=i\left(\begin{array}{cc}
-E_{2} & 0 \\
0 & E_{2}
\end{array}\right), \quad T_{3}=\left(\begin{array}{cc}
0 & E_{2} \\
-E_{2} & 0
\end{array}\right) \\
T_{4}=i\left(\begin{array}{cccc}
0 & 0 & 1 & 0 \\
0 & 0 & 0 & -1 \\
1 & 0 & 0 & 0 \\
0 & -1 & 0 & 0
\end{array}\right), \quad T_{5}=\left(\begin{array}{cccc}
0 & 0 & 0 & 1 \\
0 & 0 & -1 & 0 \\
0 & 1 & 0 & 0 \\
-1 & 0 & 0 & 0
\end{array}\right) \\
T_{6}=i\left(\begin{array}{llll}
0 & 0 & 0 & 1 \\
0 & 0 & 1 & 0 \\
0 & 1 & 0 & 0 \\
1 & 0 & 0 & 0
\end{array}\right) .
\end{gathered}
$$


Proof. It can be easily seen that the above $\left\{T_{k}\right\}_{1 \leq k \leq 6}$ is a system satisfying (4.6) with $\left(n, m_{1}, m_{2}\right)=(6,4,4)$. Conversely let $\left\{S_{k}\right\}_{1 \leq k \leq 6}$ be a system satisfying (4.6) with $\left(n, m_{1}, m_{2}\right)=(6,4,4)$. Then, by (4.6) $S_{k}$ belongs to $U(4)(1 \leq k \leq 6)$.

Now we will prove that $\left\{S_{k}\right\}_{1 \leq k \leq 6}$ is equivalent to $\left\{T_{k}\right\}_{1 \leq k \leq 6}$. Since $\left\{S_{1}, S_{2}\right\}$ is a system satisfying (4.6) with $\left(n, m_{1}, m_{2}\right)=(2,4,4)$, it follows from Lemma 5.1 that there exists a triple $\left(O_{1}, U_{1}, U_{2}\right)$ in $O(2) \times U(4) \times U(4)$ such that

$$
U_{1}\left(S_{1}, S_{2}\right)\left(O_{1} \otimes U_{2}\right)=\left(E_{4}, S_{2}^{\prime}\right),
$$

where $S_{2}^{\prime}=i E_{4}, i\left(\begin{array}{cc}-1 & 0 \\ 0 & E_{3}\end{array}\right)$ or $i\left(\begin{array}{cc}-E_{2} & 0 \\ 0 & E_{2}\end{array}\right)$. Putting $O_{2}=\left(\begin{array}{ll}O_{1} & 0 \\ 0 & E_{4}\end{array}\right) \in O(6)$, by (5.3) we have $U_{1}\left(S_{1}, \cdots, S_{6}\right)\left(O_{2} \otimes U_{2}\right)=\left(E_{4}, S_{2}^{\prime}, U_{1} S_{3} U_{2}, \cdots, U_{1} S_{6} U_{2}\right)$. So, without loss of generality we can assume that $\left(S_{1}, \ldots, S_{6}\right)=\left(E_{4}, S_{2}, \ldots\right.$, $\left.S_{6}\right)$, where $S_{2}=i E_{4}$ or $i\left(\begin{array}{cc}-1 & 0 \\ 0 & E_{3}\end{array}\right)$ or $i\left(\begin{array}{cc}-E_{2} & 0 \\ 0 & E_{2}\end{array}\right)$. The case $S_{2}=i E_{4}$ or $i\left(\begin{array}{cc}-1 & 0 \\ 0 & E_{3}\end{array}\right)$ does not occur. In fact, suppose that $S_{2}=i E_{4}$. Then it can be seen that $\left\{E_{4}, i E_{4}, S_{3}\right\}$ does not satisfy the condition (4.6). Furthermore suppose that $S_{2}=i\left(\begin{array}{cc}-1 & 0 \\ 0 & E_{3}\end{array}\right)$. Then it follows from the condition ${ }^{t} \bar{S}_{3} S_{k}+{ }^{t} \bar{S}_{k} S_{3}=0(k=1,2)$ that $S_{3}$ is represented as

$$
S_{3}=\left(\begin{array}{cccc}
0 & z_{1} & z_{2} & z_{3} \\
-\bar{z}_{1} & 0 & 0 & 0 \\
-\bar{z}_{2} & 0 & 0 & 0 \\
-\bar{z}_{3} & 0 & 0 & 0
\end{array}\right), z_{k} \in C \quad(1 \leq k \leq 3) .
$$

This contradicts to the condition ${ }^{t} \bar{S}_{3} S_{3}=E_{4}$. Hence $S_{2}$ must be $T_{2}=$ $i\left(\begin{array}{cc}-E_{2} & 0 \\ 0 & E_{2}\end{array}\right)$. From (4.6) it follows that $S_{k}(3 \leq k \leq 6)$ is represented as

$$
S_{k}=\left(\begin{array}{cc}
0 & X_{k} \\
-{ }^{t} \bar{X}_{k} & 0
\end{array}\right),{ }^{t} \bar{X}_{k} X_{l}+{ }^{t} \bar{X}_{l} X_{k}=2 \delta_{k l} E_{2} \quad(3 \leq k, l \leq 6) .
$$

We will show that $\left\{S_{k}\right\}_{1 \leq k \leq 6}$ is equivalent to $\left\{S_{k}^{\prime \prime}\right\}_{1 \leq k \leq 6}$, where $S_{1}^{\prime \prime}=T_{1}$, $S_{2}^{\prime \prime}=T_{2}$ and $S_{3}^{\prime \prime}=T_{3}$. In fact, let $U_{3}=\left(\begin{array}{cc}t \bar{X}_{3} & 0 \\ 0 & E_{2}\end{array}\right)$. Then by (5.4) we have $U_{3} \in U(4)$ and

$$
\begin{aligned}
U_{3}\left(S_{1}, \cdots, S_{6}\right)\left(E_{6} \otimes{ }^{t} \bar{U}_{3}\right) & =\left(U_{3} S_{1}{ }^{t} \bar{U}_{3}, \cdots, U_{3} S_{6}{ }^{t} \bar{U}_{3}\right) \\
& =\left(T_{1}, T_{2}, T_{3}, U_{3} S_{4}{ }^{t} \bar{U}_{3}, U_{3} S_{5}{ }^{t} \bar{U}_{3}, U_{3} S_{6}{ }^{t} \bar{U}_{3}\right) .
\end{aligned}
$$


Thus, without loss of generality we can assume that

$$
\left\{S_{k}\right\}_{1 \leq k \leq 6}=\left\{T_{1}, T_{2}, T_{3}, S_{4}, S_{5}, S_{6}\right\},
$$

where $S_{k}(4 \leq k \leq 6)$ is represented as follows;

$$
\begin{aligned}
& S_{k}=\left(\begin{array}{cr}
0 & Y_{k} \\
Y_{k} & 0
\end{array}\right), \quad{ }^{t} \bar{Y}_{k}=-Y_{k} \in U(2), \quad Y_{k} Y_{l}+Y_{l} Y_{k}=0 \\
& (4 \leq k \neq l \leq 6) .
\end{aligned}
$$

In view of (5.5) there exists $U_{4} \in U(2)$ such that $U_{4} Y_{4}{ }^{t} \bar{U}_{4}=i E_{2}$ or $-i E_{2}$ or $i\left(\begin{array}{cc}1 & 0 \\ 0 & -1\end{array}\right)$. Furthermore from the condition $Y_{4} Y_{5}+Y_{5} Y_{4}=0$ it follows that $\left(U_{4} Y_{4}{ }^{t} \bar{U}_{4}\right)\left(U_{4} Y_{5}{ }^{t} \bar{U}_{4}\right)+\left(U_{4} Y_{5}{ }^{t} \bar{U}_{4}\right)\left(U_{4} Y_{4}{ }^{t} \bar{U}_{4}\right)=0$. Therefore by the fact $U_{4} Y_{5}{ }^{t} \bar{U}_{4} \in U(2), U_{4} Y_{4}{ }^{t} \bar{U}_{4}$ must be $i\left(\begin{array}{cc}1 & 0 \\ 0 & -1\end{array}\right)$. Putting $U_{5}=$ $\left(\begin{array}{cc}U_{4} & 0 \\ 0 & U_{4}\end{array}\right) \in U(4)$, we have

$$
U_{5}\left(S_{1}, \cdots S_{6}\right)\left(E_{6} \otimes{ }^{t} \bar{U}_{5}\right)=\left(T_{1}, T_{2}, T_{3}, T_{4}, T_{5}^{\prime}, T_{6}^{\prime}\right),
$$

where $T_{5}^{\prime}$ and $T_{6}^{\prime}$ are represented as follows;

$$
T_{k}^{\prime}=\left(\begin{array}{rr}
0 & Z_{k} \\
Z_{k} & 0
\end{array}\right), \quad{ }^{t} \bar{Z}_{k}=-Z_{k} \in U(2)(k=5,6), \quad Z_{5} Z_{6}+Z_{6} Z_{5}=0 .
$$

On the other hand, by the condition ${ }^{t} \bar{T}_{4} T_{k}^{\prime}+{ }^{t} \bar{T}_{k}^{\prime} T_{4}=0(k=5,6), Z_{k}$ is represented as

$$
Z_{5}=\left(\begin{array}{cc}
0 & e^{i \theta} \\
-e^{-i \theta} & 0
\end{array}\right), \quad Z_{6}=\left(\begin{array}{cc}
0 & e^{i \eta} \\
-e^{-i \eta} & 0
\end{array}\right) \quad(\theta, \eta \in R)
$$

And by the condition $Z_{5} Z_{6}+Z_{6} Z_{5}=0$ we have $e^{i(\eta-\theta)}=\varepsilon i, \varepsilon= \pm 1$. Now we put

$$
U_{6}=\left(\begin{array}{llll}
e^{i \theta} & 0 & 0 & 0 \\
0 & 1 & 0 & 0 \\
0 & 0 & e^{i \theta} & 0 \\
0 & 0 & 0 & 1
\end{array}\right) \in U(4) \text { and } O_{3}=\left(\begin{array}{ll}
E_{5} & 0 \\
0 & \varepsilon
\end{array}\right) \in O(6)
$$

Then the direct verification shows that

$$
{ }^{t} \bar{U}_{6}\left(T_{1}, T_{2}, T_{3}, T_{4}, T_{5}^{\prime}, T_{6}^{\prime}\right)\left(O_{3} \otimes U_{6}\right)=\left(T_{1}, \cdots, T_{6}\right) .
$$

Hence, $\left\{S_{k}\right\}_{1 \leq k \leq 6}$ is equivalent to $\left\{T_{k}\right\}_{1 \leq k \leq 6}$.

q.e.d. 
5.2. We will investigate infinitesimal automorphisms of homogeneous Siegel domains over circular cones. The same notations as in the previous sections will be employed.

LEMMA 5.3. Let $D(C(n+2), F)$ be a homogeneous Siegel domain which corresponds to the skeleton $\mathbb{S}_{2}$. Then the representation $\rho$ is irreducible if and only if $m_{1}=m_{2}$ in $\mathfrak{\Im}_{2}$.

Proof. As is known in Theorem 4.4, the $C(n+2)$-hermitian form $F={ }^{t}\left(F^{1}, \cdots, F^{n+2}\right)$ is given by (4.16).

Suppose that $m_{1}=m_{2}$ in $\mathfrak{\subseteq}_{2}$. Then it was proved by PjateckiiSapiro ([10], Chap. 5, §18) that $\rho\left(g_{0}\right)$ coincides with $g(C(n+2))$. Since $C(n+2)$ is an irreducible homogeneous self-dual cone (cf. Vinberg [17]), $\mathfrak{g}(C(n+2))$ is irreducible (cf. Rothaus [11]). Thus it follows that $\rho$ is irreducible.

Now we will show that if $m_{1} \neq m_{2}$ in $\widetilde{S}_{2}$, then $\rho$ is not irreducible. It is known in [17] that the Lie algebra $g(C(n+2))$ consists of all matrices $A$ of the form;

$$
A=\left(\begin{array}{ccccc}
\lambda & 0 & 2 a_{1} & \ldots & 2 a_{n} \\
0 & \mu & 2 b_{1} & \cdots & 2 b_{n} \\
b_{1} & a_{1} & & \\
\vdots & \vdots & \frac{1}{2}(\lambda+\mu) E_{n}+\alpha \\
b_{n} & a_{n} &
\end{array}\right),
$$

where $\lambda, \mu, a_{k}$ and $b_{k}$ are real numbers $(1 \leq k \leq n)$ and $\alpha$ is a real skewsymmetric matrix of degree $n$. Let $A \in \mathfrak{g}(C(n+2))$ and $B \in \mathfrak{g l}(W)$. Then $(A, B)$ satisfies the condition; $A F(u, u)=F(B u, u)+F(u, B u)$ (for every $\left.u \in W=C^{m_{1}}+C^{m_{2}}\right)$ if and only if $B$ is represented as follows;

$$
B=\left(\begin{array}{cc}
B_{1}+\frac{1}{2} \lambda E_{m_{1}} & B_{12} \\
B_{21} & B_{2}+\frac{1}{2} \mu E_{m_{2}}
\end{array}\right),
$$

where $B_{12}=\sum a_{k} T_{k}, B_{21}=\sum b_{k}{ }^{t} \bar{T}_{k}$ and $B_{1}$ (resp. $B_{2}$ ) is a skew-hermitian matrix of degree $m_{1}$ (resp. $m_{2}$ ) satisfying the conditions

$$
B_{1}\left(T_{1}, \cdots, T_{n}\right)=\left(T_{1}, \cdots, T_{n}\right)\left(\alpha \otimes E_{m_{2}}+E_{n} \otimes B_{2}\right)
$$

and

$$
2 b_{k} E_{m_{1}}=T_{k} B_{21}+{ }^{t} \bar{B}_{21}{ }^{t} \bar{T}_{k} \quad(1 \leq k \leq n) .
$$


Now we suppose that $m_{1} \neq m_{2}$. Then by (5.9) we have

$$
2 b_{k} E_{m_{1}}=\sum_{1 \leq l \leq n} b_{l}\left(T_{k}{ }^{t} \bar{T}_{l}+T_{l}{ }^{t} \bar{T}_{k}\right) \quad(1 \leq k \leq n)
$$

From the fact ${ }^{t} \bar{T}_{k} T_{k}=E_{m_{2}}$ (cf. (4.6)) it follows that there exists $U \in U\left(m_{1}\right)$ satisfying $U T_{k}=\left(\begin{array}{c}E_{m_{2}} \\ 0\end{array}\right)$. By putting $U T_{l}=\left(\begin{array}{l}C_{l} \\ D_{l}\end{array}\right)(l \neq k)$, we have

$$
\begin{aligned}
2 b_{k} E_{m_{1}} & =\sum_{l} b_{l} U\left(T_{k}{ }^{t} \bar{T}_{l}+T_{l}{ }^{t} \bar{T}_{k}\right)^{t} \bar{U}=\sum_{l} b_{l}\left\{\left(\begin{array}{c}
E_{m_{2}} \\
0
\end{array}\right)\left({ }^{t} \bar{C}_{l},{ }^{t} \bar{D}_{l}\right)+\left(\begin{array}{c}
C_{l} \\
D_{l}
\end{array}\right)\left(E_{m_{2}}, 0\right)\right\} \\
& =\sum_{l} b_{l}\left(\begin{array}{cc}
C_{l}+{ }^{t} \bar{C}_{l} & { }^{t} \bar{D}_{l} \\
D_{l} & 0
\end{array}\right) \quad(1 \leq k \leq n),
\end{aligned}
$$

which implies that $b_{1}=b_{2}=\cdots=b_{n}=0$. From (1.7) we conclude that if $m_{1} \neq m_{2}$, then the representation $\rho$ is not irreducible. q.e.d.

The following theorem is stated implicitly in Pjateckii-Sapiro [10], as we remarked in the introduction.

THEOREM 5.4. The exceptional bounded symmetric domain in $C^{16}$ of type $(V)$ (in the sense of $\mathrm{E}$. Cartan) is realized as $D(C(8), F)$, where $F={ }^{t}\left(F^{1}, \cdots, F^{8}\right)$ is the following $C(8)$-hermitian form on $C^{8}$;

$$
\begin{aligned}
& F^{1}(u, u)=\sum_{1 \leq k \leq 4}\left|u_{k}\right|^{2}, \quad F^{2}(u, u)=\sum_{1 \leq k \leq 4}\left|u_{k+4}\right|^{2}, \\
& F^{3}(u, u)=\operatorname{Re}\left(u_{1} \bar{u}_{5}+u_{2} \bar{u}_{6}+u_{3} \bar{u}_{7}+u_{4} \bar{u}_{8}\right) \\
& F^{4}(u, u)=\operatorname{Im}\left(-u_{1} \bar{u}_{5}-u_{2} \bar{u}_{6}+u_{3} \bar{u}_{7}+u_{4} \bar{u}_{8}\right) \\
& F^{5}(u, u)=\operatorname{Re}\left(u_{1} \bar{u}_{7}+u_{2} \bar{u}_{8}-u_{3} \bar{u}_{5}-u_{4} \bar{u}_{6}\right) \\
& F^{6}(u, u)=\operatorname{Im}\left(u_{1} \bar{u}_{7}-u_{2} \bar{u}_{8}+u_{3} \bar{u}_{5}-u_{4} \bar{u}_{6}\right) \\
& F^{7}(u, u)=\operatorname{Re}\left(u_{1} \bar{u}_{8}-u_{2} \bar{u}_{7}+u_{3} \bar{u}_{6}-u_{4} \bar{u}_{5}\right) \\
& F^{8}(u, u)=\operatorname{Im}\left(u_{1} \bar{u}_{8}+u_{2} \bar{u}_{7}+u_{3} \bar{u}_{6}+u_{4} \bar{u}_{5}\right)
\end{aligned}
$$

for $u={ }^{t}\left(u_{1}, \cdots, u_{8}\right) \in C^{8}$.

Proof. We will show that the Lie algebra $g_{h}$ of all infinitesimal automorphisms of $D(C(8), F)$ is simple. It can be seen that $D(C(8), F)$ is constructed by the system $\left\{T_{k}\right\}_{1 \leq k \leq 6}$ of (5.2) by using (4.16). Thus, $D(C(8), F)$ corresponds to the skeleton $\mathfrak{S}_{2}$ with $\left(n, m_{1}, m_{2}\right)=(6,4,4)$. Therefore, by Lemma 5.3 the representation $\rho$ is irreducible.

Now we want to determine $g_{0}$. We define $A \in g(C(8))$ by putting 


$$
A=\left(\begin{array}{ccccc}
\lambda & 0 & 2 a_{1} & \ldots & 2 a_{6} \\
0 & \mu & 2 b_{1} & \cdots & 2 b_{6} \\
b_{1} & a_{1} & & \\
\vdots & \vdots & \frac{1}{2}(\lambda+\mu) E_{6}+\alpha \\
b_{6} & a_{6} &
\end{array}\right), \quad \alpha=\left(\alpha_{k l}\right) \in \mathfrak{g l}(6, R), \quad{ }^{t} \alpha=-\alpha
$$

Then by direct computations making use of (5.7), (5.8) and (5.9) we can verify that $B \in \operatorname{gr}(8, C)$ satisfies the condition; $A F(u, u)=F(B u, u)+F(u, B u)$ (for every $u \in C^{8}$ ) if and only if $B$ is represented as follows;

$$
B=\left(\begin{array}{cc}
B_{1}+\frac{1}{2} \lambda E_{4} & \sum_{1 \leq k \leq 6} a_{k} T_{k} \\
\sum_{1 \leq k \leq 6} b_{k}{ }^{t} \bar{T}_{k} & B_{2}+\frac{1}{2} \mu E_{4}
\end{array}\right)+i \theta E_{8},
$$

where $\theta \in \boldsymbol{R}$, and $B_{1}=\left(a_{\alpha \beta}\right)$ and $B_{2}=\left(b_{\alpha \beta}\right)$ are skew-hermitian matrices of degree 4 given by

$$
\begin{aligned}
& a_{12}=b_{12}=\frac{1}{2}\left\{\left(-\alpha_{35}+\alpha_{46}\right)-i\left(\alpha_{36}+\alpha_{45}\right)\right\}, \\
& a_{13}=-\bar{b}_{24}=\frac{1}{2}\left\{-\left(\alpha_{13}+\alpha_{24}\right)-i\left(\alpha_{14}-\alpha_{23}\right)\right\}, \\
& a_{14}=\bar{b}_{23}=\frac{1}{2}\left\{-\left(\alpha_{15}+\alpha_{26}\right)-i\left(\alpha_{16}-\alpha_{25}\right)\right\}, \\
& a_{23}=\bar{b}_{14}=\frac{1}{2}\left\{\left(\alpha_{15}-\alpha_{26}\right)-i\left(\alpha_{16}+\alpha_{25}\right)\right\} \\
& a_{24}=-\bar{b}_{13}=\frac{1}{2}\left\{\left(-\alpha_{13}+\alpha_{24}\right)+i\left(\alpha_{14}+\alpha_{23}\right)\right\}, \\
& a_{34}=b_{34}=\frac{1}{2}\left\{\left(\alpha_{35}+\alpha_{46}\right)+i\left(\alpha_{36}-\alpha_{45}\right)\right\}, \\
& a_{11}=i \alpha_{12}, \quad a_{22}=i\left(\alpha_{12}+\alpha_{34}+\alpha_{56}\right), \quad a_{33}=i \alpha_{34}, \quad a_{44}=i \alpha_{56}, \\
& b_{11}=0, \quad b_{22}=i\left(\alpha_{34}+\alpha_{56}\right), \quad b_{33}=i\left(\alpha_{12}+\alpha_{34}\right), \quad b_{44}=i\left(\alpha_{12}+\alpha_{56}\right) .
\end{aligned}
$$

Hence, from this fact and (1.4) it follows that $\operatorname{dim} g_{0}=\operatorname{dim} g(C(8))+1$ $=30$.

We want to show that $g_{1 / 2} \neq(0)$. We define a polynomial vector field $X=\sum_{1 \leq k \leq 8} p_{1,1}^{k} \partial / \partial z_{k}+\sum_{1 \leq \alpha \leq 8}\left(p_{1,0}^{\alpha}+p_{0,2}^{\alpha}\right) \partial / \partial w_{\alpha}$ on $C^{18}$ as follows;

$$
\begin{aligned}
& p_{1,1}^{1}=2 z_{1} w_{1}, \quad p_{1,1}^{2}=2\left\{\left(z_{3}-i z_{4}\right) w_{5}+\left(z_{5}+i z_{6}\right) w_{7}+\left(z_{7}+i z_{8}\right) w_{8}\right\} \\
& p_{1,1}^{3}=z_{1} w_{5}+\left(z_{3}-i z_{4}\right) w_{1}+\left(z_{5}+i z_{6}\right) w_{3}+\left(z_{7}+i z_{8}\right) w_{4}, \\
& p_{1,1}^{4}=-i z_{1} w_{5}+\left(i z_{3}+z_{4}\right) w_{1}+\left(-i z_{5}+z_{6}\right) w_{3}+\left(-i z_{7}+z_{8}\right) w_{4}, \\
& p_{1,1}^{5}=z_{1} w_{7}+\left(-z_{3}+i z_{4}\right) w_{3}+\left(z_{5}+i z_{6}\right) w_{1}+\left(z_{7}+i z_{8}\right) w_{2} \\
& p_{1,1}^{6}=i z_{1} w_{7}+\left(-i z_{3}-z_{4}\right) w_{3}+\left(-i z_{5}+z_{6}\right) w_{1}+\left(i z_{7}-z_{8}\right) w_{2} \\
& p_{1,1}^{7}=z_{1} w_{8}+\left(-z_{3}+i z_{4}\right) w_{4}+\left(-z_{5}-i z_{6}\right) w_{2}+\left(z_{7}+i z_{8}\right) w_{1} \\
& p_{1,1}^{8}=i z_{1} w_{8}+\left(-i z_{3}-z_{4}\right) w_{4}+\left(-i z_{5}+z_{6}\right) w_{2}+\left(-i z_{7}+z_{8}\right) w_{1},
\end{aligned}
$$

and 


$$
\begin{aligned}
& p_{1,0}^{1}=i z_{1}, \quad p_{1,0}^{2}=p_{1,0}^{3}=p_{1,0}^{4}=0, \quad p_{1,0}^{5}=i z_{3}-z_{4}, \\
& p_{1,0}^{6}=0, \quad p_{1,0}^{7}=i z_{5}+z_{6}, \quad p_{1,0}^{8}=i z_{7}+z_{8}, \\
& p_{0,2}^{1}=2 w_{1}^{2}, \quad p_{0,2}^{2}=2 w_{1} w_{2}, \quad p_{0,2}^{3}=2 w_{1} w_{3}, \\
& p_{0,2}^{4}=2 w_{1} w_{4}, \quad p_{0,2}^{5}=2 w_{1} w_{5}, \\
& p_{0,2}^{6}=2\left(w_{2} w_{5}+w_{3} w_{8}-w_{4} w_{7}\right), \quad p_{0,2}^{7}=2 w_{1} w_{7}, \quad p_{0,2}^{8}=2 w_{1} w_{8} .
\end{aligned}
$$

Then by elementary calculations, for each $c={ }^{t}\left(c^{1}, \cdots, c^{8}\right) \in C^{8}$ we have

$$
\left[\varphi_{-1 / 2}(c), X\right]=\sum a_{k l}^{\prime} z_{l} \partial / \partial z_{k}+\sum b_{\alpha \beta}^{\prime} w_{\beta} \partial / \partial w_{\alpha},
$$

where the matrices $A(c)=\left(a_{k l}^{\prime}\right)$ and $B(c)=\left(b_{\alpha \beta}^{\prime}\right)$ are given by $A(c)=$

$2\left(\begin{array}{cccccccc}2 \operatorname{Re} c^{1} & 0 & 0 & 0 & 0 & 0 & 0 & \multicolumn{1}{c}{0} \\ 0 & 0 & 2 \operatorname{Re} c^{5} & 2 \operatorname{Im} c^{5} & 2 \operatorname{Re} c^{7} & -2 \operatorname{Im} c^{7} & 2 \operatorname{Re} c^{8} & -2 \operatorname{Im} c^{8} \\ \operatorname{Re} c^{5} & 0 & \operatorname{Re} c^{1} & \operatorname{Im} c^{1} & \operatorname{Re} c^{3} & -\operatorname{Im} c^{3} & \operatorname{Re} c^{4} & -\operatorname{Im} c^{4} \\ \operatorname{Im} c^{5} & 0 & -\operatorname{Im} c^{1} & \operatorname{Re} c^{1} & \operatorname{Im} c^{3} & \operatorname{Re} c^{3} & \operatorname{Im} c^{4} & \operatorname{Re} c^{4} \\ \operatorname{Re} c^{7} & 0 & -\operatorname{Re} c^{3} & -\operatorname{Im} c^{3} & \operatorname{Re} c^{1} & -\operatorname{Im} c^{1} & \operatorname{Re} c^{2} & -\operatorname{Im} c^{2} \\ -\operatorname{Im} c^{7} & 0 & \operatorname{Im} c^{3} & -\operatorname{Re} c^{3} & \operatorname{Im} c^{1} & \operatorname{Re} c^{1} & -\operatorname{Im} c^{2} & -\operatorname{Re} c^{2} \\ \operatorname{Re} c^{8} & 0 & -\operatorname{Re} c^{4} & -\operatorname{Im} c^{4} & -\operatorname{Re} c^{2} & \operatorname{Im} c^{2} & \operatorname{Re} c^{1} & -\operatorname{Im} c^{1} \\ -\operatorname{Im} c^{8} & 0 & \operatorname{Im} c^{4} & -\operatorname{Re} c^{4} & \operatorname{Im} c^{2} & \operatorname{Re} c^{2} & \operatorname{Im} c^{1} & \operatorname{Re} c^{1}\end{array}\right)$,

$$
B(c)=2\left(\begin{array}{rrrrrccc}
c^{1} & -\overline{c^{2}} & -\overline{c^{3}} & -\overline{c^{4}} & 0 & 0 & 0 & 0 \\
c^{2} & \overline{c^{1}} & 0 & 0 & 0 & 0 & 0 & 0 \\
c^{3} & 0 & \overline{c^{1}} & 0 & 0 & 0 & 0 & 0 \\
c^{4} & 0 & 0 & \overline{c^{1}} & 0 & 0 & 0 & 0 \\
c^{5} & 0 & -\overline{c^{7}} & -\overline{c^{8}} & 0 & -\overline{c^{2}} & 0 & 0 \\
0 & c^{5} & c^{8} & -c^{7} & c^{2} & -2 i \operatorname{Im} c^{1} & -c^{4} & c^{3} \\
c^{7} & -\overline{c^{8}} & \overline{c^{5}} & 0 & 0 & \overline{c^{4}} & 0 & 0 \\
c^{8} & \overline{c^{7}} & 0 & \overline{c^{5}} & 0 & -\overline{c^{3}} & 0 & 0
\end{array}\right)+4 i \operatorname{Im} c^{1} E_{8} .
$$

Hence by (5.6), $A(c)$ belongs to $g(C(8))$. Considering (5.11) we can verify that $(A(c), B(c))$ satisfies the condition; $A(c) F(u, u)=F(B(c) u, u)+$ $F(u, B(c) u)$ for every $u \in C^{8}$. Therefore, by (1.4) $\left[\varphi_{-1 / 2}(c), X\right]$ belongs to $\mathfrak{g}_{0}$, and we have $\left[\mathfrak{g}_{-1 / 2}, X\right] \subset \mathfrak{g}_{0}$. From (1.9), thus it follows that $X$ belongs to $\mathfrak{g}_{1 / 2}$ and $\mathfrak{g}_{1 / 2} \neq(0)$.

So, as a consequence of Theorem 2.1, we conclude that $\mathfrak{g}_{h}$ is simple. By the well-known theorem of Borel-Koszul [1], [7], $D(C(8), F)$ is holo- 
morphically isomorphic to an irreducible bounded symmetric domain in $C^{16}$.

This bounded symmetric domain is the exceptional domain of type $(V)$. In fact, by using (1.6) we have $\operatorname{dim} \mathfrak{g}_{h}=2\left(\operatorname{dim} \mathfrak{g}_{-1}+\operatorname{dim} \mathfrak{g}_{-1 / 2}\right)+$ $\operatorname{dim} \mathfrak{g}_{0}=78$. And there is no classical irreducible bounded symmetric domain in $C^{16}$ whose Lie algebra of all infinitesimal automorphisms is of dimension 78 (cf. e.g., Helgason [2]).

q.e.d.

Remark. The form $F$ given by (5.10) is different from that of the note [15]. But it can be seen that this domain is isomorphic to that of [15] under a linear transformation (cf. Proposition 5.2).

\section{§ 6. Automorphisms of Siegel domains over circular cones}

In this section, we calculate infinitesimal automorphisms of homogeneous Siegel domains over circular cones.

The Lie algebra $g_{h}$ of a homogeneous non-degenerate Siegel domain $D(C(n+2), F)$ for which the representation $\rho$ is irreducible is determined completely by the following theorem.

THEOREM 6.1. The Lie algebra $\mathfrak{g}_{h}$ of all infinitesimal automorphisms of a homogeneous Siegel domain $D\left(C(n+2), F^{\prime}\right)$ which corresponds to the skeleton $\mathfrak{S}_{2}$ with $m_{1}=m_{2}(=m)$ is given as follows;

\begin{tabular}{|c|l|}
\hline$(n, m)$ & \multicolumn{1}{|c|}{$\mathrm{g}_{h}$} \\
\hline & (i) $\mathrm{g}_{h}=\mathfrak{g}_{\mathfrak{H}}(m+2,2)$ provided that $D\left(C(4), F^{\prime}\right)$ is constructed \\
$(2, m)$ & by the system $\left\{T_{1}, T_{2}\right\} \quad\left(T_{1}, T_{2} \in U(m)\right)$ such that $\bar{T}_{1} T_{2}$ has \\
& $\begin{array}{l}\{i, \cdots, i\} \text { or }\{-i, \cdots,-i\} \text { as its eigenvalues. } \\
\text { (ii) } \mathrm{g}_{h}=\mathrm{g}_{a}, \text { otherwise. }\end{array}$ \\
\hline$(4,2)$ & $\mathrm{g}_{h}=\mathfrak{g}_{0} *(10)$ \\
\hline$(6,4)$ & $\mathrm{g}_{h}=\mathrm{e}_{6}(-14)$ \\
\hline otherwise & $\mathfrak{g}_{h}=\mathfrak{g}_{a}$ \\
\hline
\end{tabular}

Proof. Pjateckii-Sapiro ([10], Chap. 2) gave case by case the explicit realizations of all classical domains. From his realizations it follows that if $D(C(n+2), F)$ is classical, then $(n, m)=(2, m)$ or $(4,2)$.

Suppose that $(n, m)=(2, m)$. Then it was proved in [10] that $D(C(4), F)$ is a symmetric domain if and only if ${ }^{t} \bar{T}_{1} T_{2}$ has $\{i, \cdots, i\}$ or 
$\{-i, \cdots,-i\}$ as its eigenvalues and that in this case $D(C(4), F)$ is the classical domain in $C^{4+2 m}$ of type (I).

Suppose that $(n, m)=(4,2)$. Then there exists a unique homogeneous Siegel domain which corresponds to the skeleton $\mathfrak{S}_{2}$ with $\left(n, m_{1}, m_{2}\right)=$ $(4,2,2)$ (cf. [10], [16]). And it was proved in [10] that this domain is the classical domain in $C^{10}$ of type (II).

Suppose that $(n, m)=(6,4)$. Then there exists a unique homogeneous Siegel domain which corresponds to the skeleton $\mathfrak{S}_{2}$ with $\left(n, m_{1}, m_{2}\right)=$ $(6,4,4)$ (Proposition 5.2) and this domain is the exceptional domain in $C^{16}$ of type (V) (Theorem 5.4).

By the uniqueness theorem of realization (cf. Kaneyuki [3]), there exists no symmetric Siegel domain of type II over circular cones other than the domains listed above (cf. [10], and for the exceptional domain of type (VI), see e.g., Vinberg [17]). Thus, our assertion follows from Theorem 2.1 and Lemma 5.3.

q.e.d.

Now we determine infinitesimal automorphisms of homogeneous degenerate Siegel domains of type II over $C(n+2)$. As we stated in section 4 , every homogeneous degenerate Siegel domain $D(C(n+2), F)$ in $C^{n+2} \times C^{m}(m>0)$ can be constructed by the following $C(n+2)$ hermitian form $\boldsymbol{F}$ on $\boldsymbol{C}^{m}$;

$$
F(u, v)={ }^{t}((u, v), 0, \cdots, 0), \quad u, v \in C^{m} \text { (cf. (4.17)) . }
$$

Proposition 6.2. For the homogeneous degenerate Siegel domain $D(C(n+2), F)$ in $C^{n+2} \times C^{m}(m>0)$, the subspaces $\mathfrak{g}_{1 / 2}$ and $\mathfrak{g}_{1}$ of $\mathfrak{g}_{h}$ are given by

$$
\begin{aligned}
\mathfrak{g}_{1 / 2} & =(0), \\
\mathfrak{g}_{1} & =\left\{a\left(\sum_{1 \leq k \leq n} z_{k+2}^{2} \partial / \partial z_{1}+z_{2}^{2} \partial / \partial z_{2}+\sum_{1 \leq k \leq n} z_{2} z_{k+2} \partial / \partial z_{k+2}\right) ; a \in \boldsymbol{R}\right\} .
\end{aligned}
$$

Proof. First we will determine $\mathrm{g}_{0}$. Let $A \in \mathfrak{g}(C(n+2))$ and $B \in \mathfrak{g l}(m, C)$. Then it can be easily verified that $(A, B)$ satisfies the condition; $A F(u, u)$ $=F(B u, u)+F(u, B u)$ (for each $\left.u \in C^{m}\right)$ if and only if $(A, B)$ is represented as

$$
A=\left(\begin{array}{ccccc}
\lambda & 0 & 2 a_{1} & \ldots & 2 a_{n} \\
0 & \mu & 0 & \ldots & 0 \\
0 & a_{1} & & \\
\vdots & \vdots & \frac{1}{2}(\lambda+\mu) E_{n}+\alpha \\
0 & a_{n} &
\end{array}\right), \quad B+{ }^{t} \bar{B}=\lambda E_{m}
$$


where $\lambda, \mu, a_{k}(1 \leq k \leq n)$ are real numbers and $\alpha$ is a real skew-symmetric matrix of degree $n$ (cf. (5.6)). Thus, by (1.4) we have determined $g_{0}$.

Now we show $g_{1 / 2}=(0)$. In view of Corollary 2.7 we can assume that $m=1$. Let $X \in g_{1 / 2}$. Then by (2.2), (2.3) and (2.4), there exist $c_{l}, b \in C(1 \leq l \leq n+2)$ satisfying the following conditions;

(6.2) $X$ is represented as $X=2 i \sum \bar{c}_{l} z_{l} w \partial / \partial z_{1}+\sum c_{l} z_{l} \partial / \partial w+b w^{2} \partial / \partial w$,

(6.3) $b=2 i \bar{c}_{1}$,

(6.4) for each $d \in C$, the matrix

$$
\left(\begin{array}{cccc}
\operatorname{Im}\left(c_{1} \bar{d}\right) & \operatorname{Im}\left(c_{2} \bar{d}\right) & \cdots & \operatorname{Im}\left(c_{n+2} \bar{d}\right) \\
0 & 0 & \cdots & 0 \\
\vdots & \vdots & & \vdots \\
0 & 0 & \cdots & 0
\end{array}\right)
$$

belongs to $g(C(n+2))$.

Hence, by (5.6) and (6.4), $\operatorname{Im}\left(c_{l} \bar{d}\right)=0$ for each $d \in C(1 \leq l \leq n+2)$. So, $c_{l}=0(1 \leq l \leq n+2)$. From (6.2) and (6.3) it follows that $X=0$. Thus, $\mathfrak{g}_{1 / 2}=(0)$ was proved.

Now we determine $g_{1}$. By (1.3) we have

$$
\mathfrak{g}_{-1 / 2}=\left\{2 i(w, c) \partial / \partial z_{1}+\sum c^{\alpha} \partial / \partial w_{\alpha} ; c=\sum c^{\alpha} f_{\alpha} \in C^{m}\right\}
$$

Let $X=\sum p_{2,0}^{k} \partial / \partial z_{k}+\sum p_{1,1}^{\alpha} \partial / \partial w_{\alpha} \in \mathfrak{g}_{1}$. Then by the condition $\left[\mathfrak{g}_{-1 / 2}, X\right]$ $=(0)$, we get $\partial p_{2,0}^{k} / \partial z_{1}=0(1 \leq k \leq n+2)$ and $p_{1,1}^{\alpha}=0(1 \leq \alpha \leq m)$. We write $p_{2,0}^{k}=\sum a_{i j}^{k} z_{i} z_{j}\left(a_{i j}^{k}=a_{j i}^{k}\right)$. Then we have

$$
a_{1 j}^{k}=a_{j 1}^{k}=0 \quad(1 \leq j, k \leq n+2) .
$$

For each $i(1 \leq i \leq n+2)$, we define the $(n+2) \times(n+2)$-matrix $A_{i}$ by

$$
A_{i}=\left(\begin{array}{cccc}
a_{i 1}^{1} & a_{i 2}^{1} & \cdots & a_{i n+2}^{1} \\
a_{i 1}^{2} & a_{i 2}^{2} & \cdots & a_{i n+2}^{2} \\
\vdots & \vdots & & \vdots \\
a_{i 1}^{n+2} & a_{i 2}^{n+2} & \cdots & a_{i n+2}^{n+2}
\end{array}\right)
$$

Then we have

$$
\frac{1}{2} \rho\left(\left[\partial / \partial z_{i}, X\right]\right)=A_{i} \text { and } \sigma\left(\left[\partial / \partial z_{i}, X\right]\right)=0 .
$$


By (1.10) and (1.4), $\left(A_{i}, 0\right)$ must be of the form (6.1). Comparing (6.6) with (6.1), we can see that the real numbers $a_{i j}^{k}(1 \leq i, j, k \leq n+2)$ must satisfy the following relations;

$$
\begin{aligned}
& a_{i k+2}^{1}=2 a_{i 2}^{k+2} \quad(1 \leq i \leq n+2,1 \leq k \leq n) \\
& a_{i 2}^{1}=0 \quad(1 \leq i \leq n+2) \\
& a_{i 2}^{2}=2 a_{i k+2}^{k+2} \quad(1 \leq i \leq n+2,1 \leq k \leq n) \\
& a_{i k+2}^{2}=0 \quad(1 \leq i \leq n+2,1 \leq k \leq n) \\
& a_{i l+2}^{k+2}=-a_{i k+2}^{l+2} \quad(1 \leq i \leq n+2,1 \leq k \neq l \leq n)
\end{aligned}
$$

By (6.5) we have $a_{i 1}^{1}=a_{1 i}^{1}=0(1 \leq i \leq n+2)$. Applying (6.7) and (6.11) for $1 \leq k \neq l \leq n$, we get

$$
a_{k+2 l+2}^{1}=2 a_{k+22}^{l+2}=2 a_{2 k+2}^{l+2}=-2 a_{2 l+2}^{k+2}=-2 a_{l+22}^{k+2}=-a_{l+2 k+2}^{1}=-a_{k+2 l+2}^{1},
$$

which implies $a_{k+2 l+2}^{1}=0$. Therefore, considering (6.8) we showed (6.12) $a_{i j}^{1}=0$ if $1 \leq i \leq 2$ or $1 \leq j \leq 2$ or $3 \leq i \neq j \leq n+2$.

By (6.5) and (6.10) we get

$$
a_{i j}^{2}=0 \quad \text { if }(i, j) \neq(2,2) \text {. }
$$

From (6.5) we have $a_{1 i}^{k+2}=a_{i 1}^{k+2}=0(1 \leq i \leq n+2)$ and by (6.7), (6.12) we can see $a_{2 i}^{k+2}=a_{i 2}^{k+2}=0 \quad(i=2$ or $3 \leq i \neq k+2 \leq n+2)$. Furthermore if $1 \leq i \neq j \neq k \neq i \leq n$, then by (6.11) $a_{i+2 j+2}^{k+2}$ is skew-symmetric with respect to the indices $j, k$ and symmetric with respect to the indices $i, j$. So, $a_{i+2 j+2}^{k+2}=0$ if $1 \leq i \neq j \neq k \neq i \leq n$. Hence by (6.9), (6.11) we have

(6.14) $a_{i j}^{k+2}=0 \quad$ if $(i, j) \neq(2, k+2)$ and $(i, j) \neq(k+2,2)(1 \leq k \leq n)$.

On the other hand, we can see

$$
\begin{aligned}
a_{22}^{2} & =2 a_{2 k+2}^{k+2} \quad(\text { by }(6.9)) \\
& =a_{k+2 k+2}^{1} \quad(\text { by }(6.7))(1 \leq k \leq n) .
\end{aligned}
$$

As a consequence of (6.12)-(6.15), it follows that $X$ must be represented by

$$
X=a_{22}^{2}\left(\sum_{1 \leq k \leq n} z_{k+2}^{2} \partial / \partial z_{1}+z_{2}^{2} \partial / \partial z_{2}+\sum_{1 \leq k \leq n} z_{2} z_{k+2} \partial / \partial z_{k+2}\right)
$$


Conversely if $X$ is a polynomial vector field of the form (6.16), then it can be easily seen that $X$ satisfies all the conditions in (1.10). Thus, the subspace $g_{1}$ of $g_{h}$ consists of all polynomial vector fields of the form (6.16).

q.e.d.

Finally we consider the homogeneous non-degenerate Siegel domains which correspond to the skeleton $\widetilde{S}_{2}$ with $n \leq 2 m_{2}<2 m_{1}$. Let $\left\{T_{k}\right\}_{1 \leq k \leq n}$ be a system of $m_{2} \times m_{2}$-matrices satisfying the condition (4.6). We put $T_{k}^{\prime}=\left(\begin{array}{c}T_{k} \\ 0\end{array}\right)$, where 0 means the $\left(m_{1}-m_{2}\right) \times m_{2}$-zero matrix. Then it is easy to see that the system $\left\{T_{k}^{\prime}\right\}_{1 \leq k \leq n}$ satisfies the condition (4.6) and corresponds to this skeleton $\widetilde{S}_{2}$. We denote by $D(C(n+2), F)$ the Siegel domain in $C^{n+2} \times C^{m_{1}+m_{2}}$ which is constructed by the system $\left\{T_{k}^{\prime}\right\}_{1 \leq k \leq n}$. Then, by (4.16) the $C(n+2)$-hermitian form $F$ is given by

$$
\begin{aligned}
& F^{1}(u, v)=\left(u_{1}, v_{1}\right)+\left(u_{3}, v_{3}\right), \quad F^{2}(u, v)=\left(u_{2}, v_{2}\right), \\
& F^{k+2}(u, v)=\frac{1}{2}\left\{\left(u_{1}, T_{k} v_{2}\right)+\left(T_{k} u_{2}, v_{1}\right)\right\} \quad(1 \leq k \leq n)
\end{aligned}
$$

for $u=\left(u_{1}+u_{3}\right)+u_{2}, v=\left(v_{1}+v_{3}\right)+v_{2} \in \boldsymbol{C}^{m_{1}+m_{2}}=\left(\boldsymbol{C}^{m_{2}}+\boldsymbol{C}^{m_{1}-m_{2}}\right)+\boldsymbol{C}^{m_{2}}$.

Proposition 6.3. For the Siegel domain $D(C(n+2), F)$ given by (6.17), if $n \neq 2,\left(n, m_{2}\right) \neq(4,2)$ and $\left(n, m_{2}\right) \neq(6,4)$, then $\mathfrak{g}_{h}=\mathfrak{g}_{a}$. If $n$ $=2$ and ${ }^{t} \bar{T}_{1} T_{2}$ does not have $\{i, \cdots, i\}$ and $\{-i, \cdots,-i\}$ as its eigenvalues, then $\mathfrak{g}_{h}=\mathfrak{g}_{a}$.

Proof. We put the subspaces $W_{1}$ and $W_{2}$ of $\boldsymbol{C}^{m_{1}+m_{2}}=\left(\boldsymbol{C}^{m_{1}}+\boldsymbol{C}^{m_{1}-m_{2}}\right)$ $+\boldsymbol{C}^{m_{2}}$ by $W_{1}=\boldsymbol{C}^{m_{2}}+\boldsymbol{C}^{m_{2}}$ and $W_{2}=C^{m_{1}-m_{2}}$, respectively. Then we can see that $F\left(W_{1}, W_{2}\right)=(0)$. The Siegel domain $D\left(C(n+2), F_{2}\right)$ in $C^{n+2} \times W_{2}$ is the one given in Proposition 6.2. Therefore we have $g_{1 / 2}^{(2)}=(0)$. On the other hand, the Siegel domain $D\left(C(n+2), F_{1}\right)$ in $C^{n+2} \times W_{1}$ is the one given in Theorem 6.1. Thus, by Theorem 6.1 we get $\mathfrak{g}_{1 / 2}^{(1)}=(0)$. From Corollary 2.7 it follows that $\mathfrak{g}_{1 / 2}=(0)$. Applying Proposition 2.2 to the non-degenerate Siegel domain $D(C(n+2), F)$, we conclude that $\mathfrak{g}_{h}=\mathfrak{g}_{a}$.

q.e.d.

\section{BIBLIOGRAPHY}

[1] A. Borel, Kählerian coset spaces of semi-simple Lie groups, Proc. Nat. Acad. Sci. U.S.A., 40 (1954), 1147-1151.

[2] S. Helgason, Differential Geometry and Symmetric Spaces, Academic Press, New York, 1962.

[ 3 ] S. Kaneyuki, On the automorphism groups of homogeneous bounded domains, J. Fac. Sci. Univ. Tokyo, 14 (1967), 89-130. 
[ 4 ] S. Kaneyuki and M. Sudo, On Silov boundaries of Siegel domains, J. Fac. Sci. Univ. Tokyo, 15 (1968), 131-146.

[5] S. Kaneyuki and T. Tsuji, Classification of homogeneous bounded domains of lower dimension, (to appear in Nagoya Math. J., 53).

[6] W. Kaup, Y. Matsushima and T. Ochiai, On the automorphisms and equivalences of generalized Siegel domains, Amer. J. Math., 92 (1970), 475-498.

[ 7 ] J. L. Koszul, Sur la forme hermitienne canonique des espaces homogènes complexes, Canad. J. Math., 7 (1955), 562-576.

[ 8 ] S. Murakami, On Automorphisms of Siegel Domains, Lecture Notes in Math., 286, Springer, 1972.

[ 9 ] K. Nakajima, Some studies on Siegel domains, (preprint).

[10] I. I. Pjateckii-Sapiro, Géométrie des Domaines Classiques et Théorie des Fonctions Automorphes, French translation, Dunod, Paris, 1966.

[11] O. S. Rothaus, Domains of positivity, Abh. Math. Sem. Univ. Hamburg, 24 (1960), 189-235.

[12] M. Sudo, On infinitesimal automorphisms of Siegel domains over classical cones, (preprint).

[13] M. Takeuchi, On infinitesimal affine automorphisms of Siegel domains, Proc. Japan Acad., 45 (1969), 590-594.

[14] N. Tanaka, On infinitesimal automorphisms of Siegel domains, J. Math. Soc. Japan, 22 (1970), 180-212.

[15] T. Tsuji, On infinitesimal automorphisms and homogeneous Siegel domains over circular cones, Proc. Japan Acad., 49 (1973), 390-393.

[16] T. Tsuji, Classification of homogeneous Siegel domains of type II of dimensions 9 and 10, Proc. Japan Acad., 49 (1973), 394-396.

[17] E. B. Vinberg, The structure of the group of automorphisms of a homogeneous convex cone, Trans. Moscow Math. Soc., 13 (1965), 63-93.

[18] K. Nakajima, Symmetric spaces associated with Siegel domains, (preprint).

Mie University 